\title{
A high-dynamic-range visual sensing method for feature extraction of welding pool based on adaptive image fusion
}

\section{Baori Zhang}

South China University of Technology

Yonghua Shi ( $\square$ yhuashi@scut.edu.cn )

South China University of Technology

Yanxin Cui

South China University of Technology

Zishun Wang

South China University of Technology

Xiyin Chen

South China University of Technology

\section{Research Article}

Keywords: Adaptive monitoring, Image fusion, Keyhole TIG welding, Welding dynamic range, Feature detection

Posted Date: March 12th, 2021

DOI: https://doi.org/10.21203/rs.3.rs-281669/v1

License: (c) (i) This work is licensed under a Creative Commons Attribution 4.0 International License. Read Full License

Version of Record: A version of this preprint was published at The International Journal of Advanced Manufacturing Technology on August 11th, 2021. See the published version at https://doi.org/10.1007/s00170-021-07812-x. 


\title{
A high-dynamic-range visual sensing method for feature extraction of welding pool based on adaptive image fusion Baori Zhang $\cdot$ Yonghua Shi* $\cdot$ Yanxin Cui $\cdot$ Zishun Wang $\cdot$ Xiyin Chen
}

Guangdong Provincial Engineering Research Center for Special Welding Technology and Equipment, School of Mechanical and Automotive Engineering, South China University of Technology, Guangzhou 510640, China

\begin{abstract}
The high dynamic range existing in arc welding with high energy density challenges most of the industrial cameras, causing badly exposed pixels in the captured images and bringing difficulty to the feature detection from internal weld pool. This paper proposes a novel monitoring method called adaptive image fusion, which increases the amount of information contained in the welding image and can be realized on the common industrial camera with low cost. It combines original images captured rapidly by the camera into one fused image and the setting of these images is based on the real time analysis of realistic scene irradiance during the welding. Experiments are carried out to find out the operating window for the adaptive image fusion method, providing the rules for getting a fused image with as much as information as possible. The comparison between the imaging with or without the proposed method proves that the fused image has a wider dynamic range and includes more useful features from the weld pool. The improvement is also verified by extracting both the internal and external features of weld pool within a same fused image with proposed method. The results show that the proposed method can adaptively expand the dynamic range of visual monitoring system with low cost, which benefits the feature extraction from the internal weld pool.
\end{abstract}

Keywords Adaptive monitoring · Image fusion · Keyhole TIG welding $\cdot$ Welding dynamic range $\cdot$ Feature detection

\section{Introduction}

High energy density welding has a wide application in the fields of industrial manufacturing because of its high efficiency[1]. The high heat input of the welding torch enables the application of a higher travelling speed or thicker welding weldment. As a variant of the traditional TIG welding, keyhole TIG welding achieves a high energy input with large current by using torch cooling system. It features with its single-pass welding of medium or high thickness metal weldments[2]. However, the arc with highly-concentrated energy releases a huge number of photons during the welding scene. The welding region under the arc has high brightness because of the heat radiance and the reflection of arc light from the metal surface[3]. It brings challenges in visual monitoring of the molten pool, causing over-exposed or under-exposed pixels in the images. In order to solve this problem. Some researchers applied advanced digital camera to obtain well-exposed images. Xia et al. used a Xiris XVC-1000e camera to get clear images of the weld pool front the back side[4]. The head and tail of the weld pool could not be captured clearly at the same time. Wang et al. applied NIT MC1003-1VB camera into getting a high dynamic range image of welding process[5]. Bakir et al. put a s-CMOS camera on a focusing optical head of a laser for better a texture recognition of the welding[6]. De, A. et al. located the weld seam ahead of the welding torch using images sensed by a simple CCD monochrome camera[7]. However, these cameras contain expensive

*Corresponding author: Yonghua Shi (yhuashi@ @scut.edu.cn) 
photosensitive elements which increases the cost of the visual system setting up. Besides, the existing circuit settings in the imaging element determines that its imaging strategy cannot be adapted to the brightness changes in the scene. Some researchers used optical systems composed of extra light source with specific frequency and narrow filters. Luo $\mathrm{M}$ et al. used a laser with green light to light up the weld region and took pictures through an filter for width detection of molten pool[8]. Shao et al. used three colors of laser to capture and process a single image so that the three dimensional position of the space weld seam can be obtained simultaneously[9]. But this method requires series of previous experiments to decide the choice of light sources and filters and would need repeat adjustment once the welding scene changes with the operating parameters. In addition, the inner features of the weld pool are hardly recorded with the methods above due to their extremely high brightness[10,11]. As a result, the visual monitoring and detection in keyhole TIG welding becomes difficult because. There is a high contrast in brightness as well as high oscillation within the weld pool. The welding quality is closely related to the internal and external geometry of the weld pool, so obtaining a panoramic view of the weld pool is crucial.

In this paper, a novel method called adaptive image fusion is proposed to solve the monitoring and detecting problem in keyhole TIG welding. It enables the low-cost camera to adaptively capture clear images of high-dynamic-range welding scene, realizing the complete features extraction of the weld pool. The section 2 analyzes the dynamic range of keyhole TIG welding by scene irradiance measurement, explaining the key reason for the existing monitoring problem. The section 3.1 designs an adaptive imaging strategy based on the real-time relative irradiance detection. Based on this, the section 3.2 introduces a novel image fusion algorithm to obtain images of keyhole TIG welding of high dynamic range. Besides, tests are also carried out to determine the operating window for the parameter configuration in image fusion. Then, section 4 realizes the detection of the overall geometry of weld pool including its external profile and the size of the keyhole entrance inside the weld pool. Finally, section 5 concludes the performance of the proposed monitoring method, showing that the image fusion algorithm can be adaptive to wide-dynamic-range keyhole TIG welding scene and can be performed on economic cameras.

\section{Dynamic range of the keyhole TIG welding scene}

The plasma arc during keyhole TIG welding process produces strong light and high heat input. Therefore, the weld pool under the arc emits great amount of light due to its heat radiance as well as the arc light reflected from the pool surface[12,13]. But the brightness of the light decreases rapidly in the direction away from the arc. It brings high contrast between different parts of the weld pool, causing the difficulty in clear image capturing during welding process. Therefore, having a complete knowledge of the brightness range of the keyhole TIG welding scene does help to design suitable strategy for visual monitoring.

In this section, dynamic range is used to describe the brightness range between the darkest and brightest parts within a realistic scene or an image. It shows the irradiance range of a scene or the ability of an imaging system to record the realistic scene[14]. Experiments are carried out on the keyhole TIG welding with different kinds of CMOS cameras. The dynamic ranges of both the welding scene and the cameras are obtained for comparison. Figure 1 illustrates the process of the measurement of the dynamic range of the welding scene. A method of calibration was used to reconstruct response function of the camera by taking weld pool images with various exposure times. First, the calibration for the response property of the camera is taken. It is described as a formula describing the relationship among the pixel value $Z$, exposure time $\Delta t$ and the irradiance $L$ in reference [15]:

$$
f(Z, \Delta t, L)=\exp (g(Z))-A \Delta t L=0
$$

Where $g(Z)$ is the response function of the camera and $A$ represents the proportionality factor, 
which is a constant and determined by the camera installation and the use of the filter. Then several welding images of the same keyhole TIG welding scene are taken with various exposure times. The next step is to get the relative irradiance map from each image captured before via formula (1). Every relative irradiance map describes a part of the welding scene. Finally, by combining these maps, a complete relative irradiance map of the keyhole TIG welding scene can be obtained. From the map, the maximum and minimum relative irradiances are taken to calculate the dynamic range (DR) of the scene through the following formula:

$$
D R=\ln \frac{L_{\max }}{L_{\min }}
$$

Where $L_{\max }$ and $L_{\min }$ stand for the maximum and minimum irradiance of the welding scene respectively. The dynamic ranges of the keyhole TIG welding scene with different currents are listed in figure 2. The figure also includes the dynamic range of the commonly used CMOS cameras in welding monitoring as well as the camera used in this study[16]. The result shows that for the commonly used CMOS camera, their dynamic range is not wide enough to cover the whole keyhole TIG welding scene. That is the reason for why the common cameras could not record complete information from the welding scene. Besides, the result also shows that the dynamic range of welding scene will change as the welding parameters changes while the dynamic range of the camera is fixed. As a result, a camera with a much larger dynamic range must be used to ensure image quality, which increases unnecessary cost. In order to solve this problem, an adaptive method is needed to improve dynamic range of the image during keyhole TIG welding monitoring with low cost.

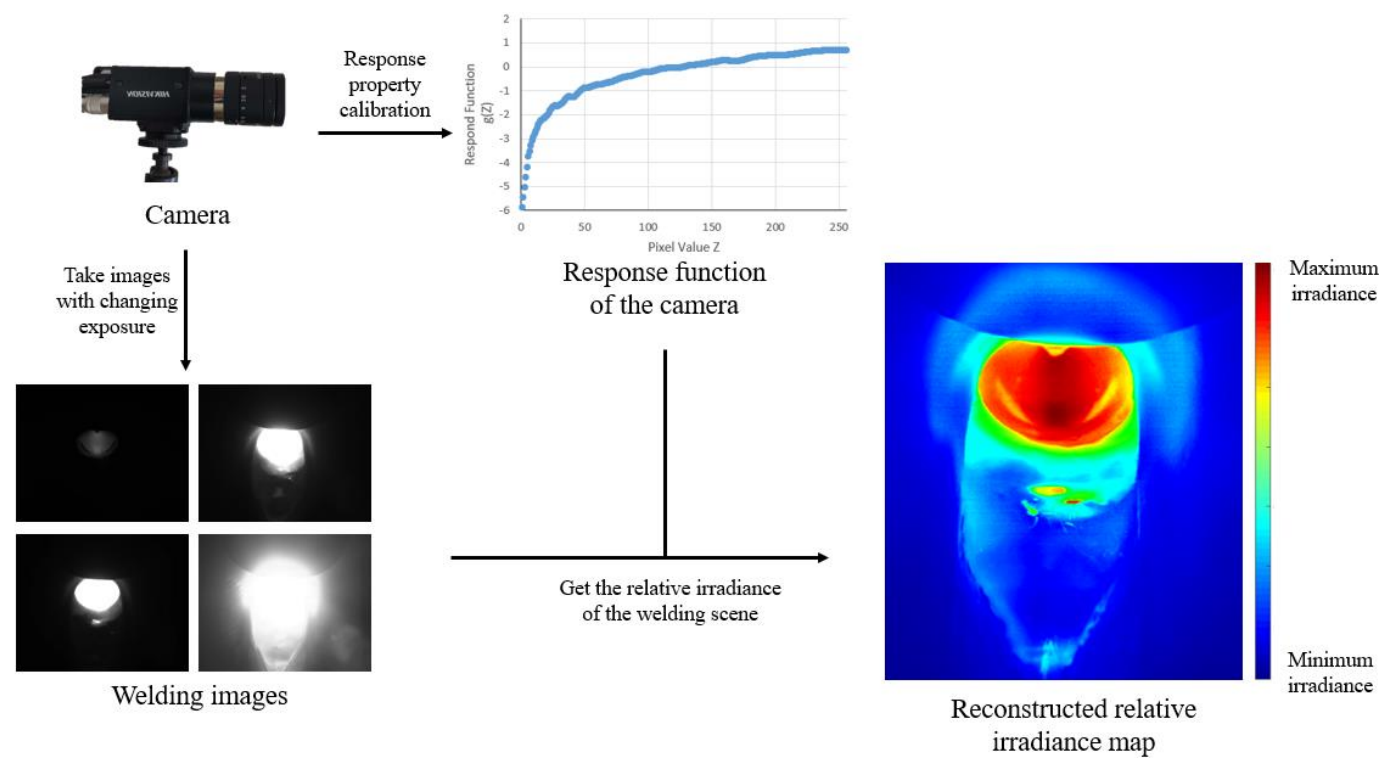

Fig. 1 The process of the measurement of the dynamic range of the welding scene 


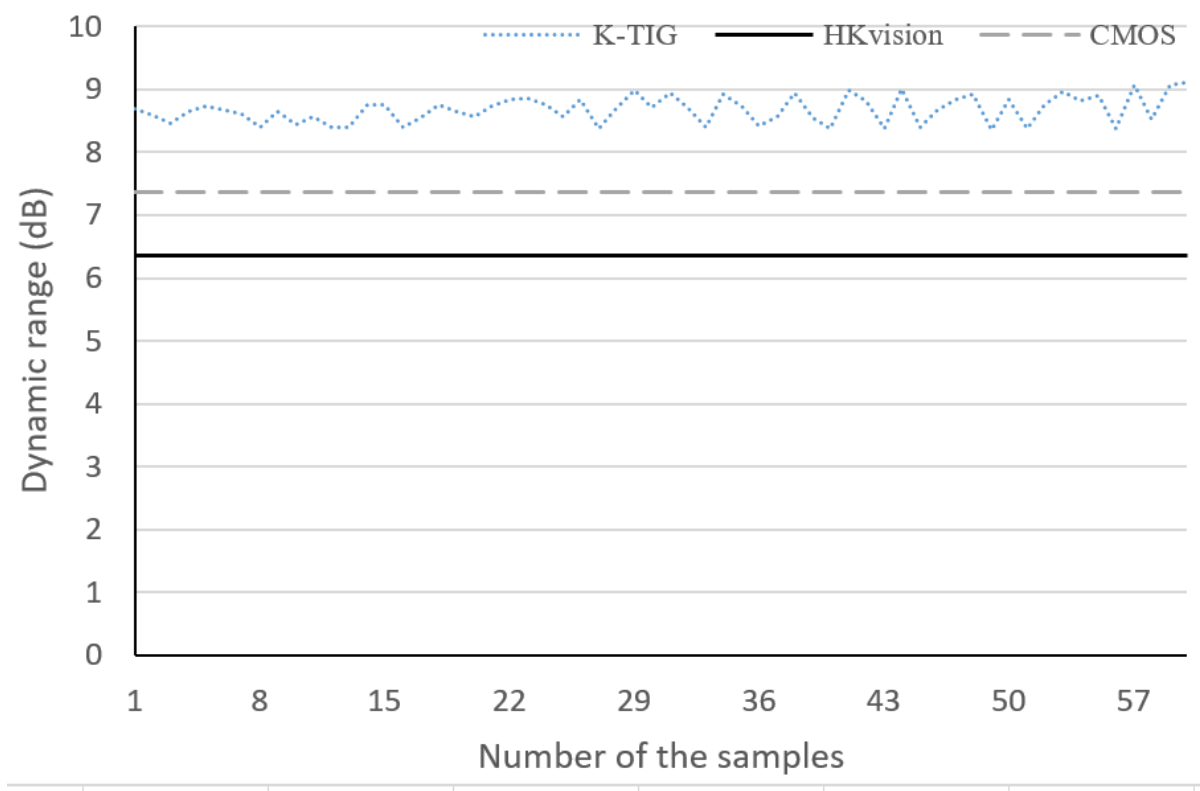

Fig. 2 The comparison of the dynamic range of the welding scene and camera

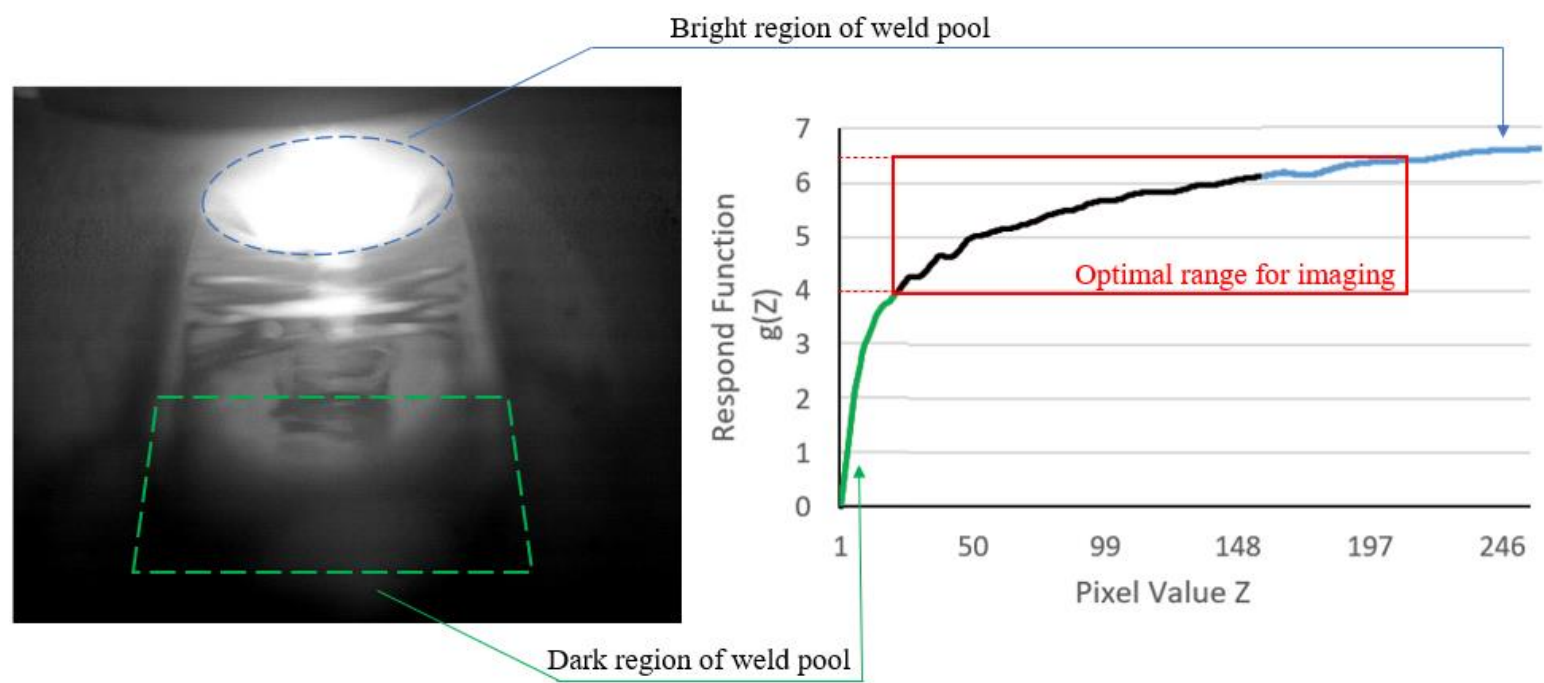

Fig. 3 Analysis of the different portion of the response function curve of the camera.

\section{Adaptive image fusion in welding monitoring}

In this section, an adaptive image fusion algorithm is proposed to improve the dynamic range of the images of keyhole TIG welding. First, a set of images will be captured periodically with various exposure times following an irradiance-based imaging rule. Then, an image fusion algorithm is used to combine these images to obtain a new image which covers the whole dynamic range of the welding scene. Finally, the influence of the camera characteristic on the performance of the proposed algorithm is analyzed through experiments. The operating window for choosing the optimal parameters of the camera are also concluded. In this way, the dynamic range of the visual system can be widened without updating the camera electronics. The quality of the new image can also be guaranteed by adjusting all the exposure time of the images in real time.

\section{1 irradiance-based imaging of weld pool}

According to the irradiance map shown in figure 1, different parts of the weld pool have different dynamic ranges. To be specific, the keyhole inside the head of the weld pool has a highest brightness, while there is a decline in irradiance from the head to the tail of the weld pool. The automatic gain control 
(AGC) strategy is commonly used in camera imaging, which depends on the statistic analysis of the pixel histogram instead of the irradiance distribution[17]. It is not suitable to record the details inside the weld pool because it has high irradiance. In addition, the way that camera transfers the irradiance to the pixel value causes problems. The response function is a logarithmic curve describing this transforming, as shown in figure 3. On one hand, the bright region of the weld pool corresponds to the blue part of the curve, which is very flat. It means that only a small portion pixel inside the weld pool can be well-exposed. On the other hand, the dark region of the weld pool corresponds to the green part of the curve, which is steep. This means that a small increase in the pixel value will cause a large change in radiance. It will lead to error of calculation. As a result, the propriate portion of the curve for mapping is the middle one that is not too flat or steep. In this paper, this portion of curve is called the optical range for imaging (ORI), as shown in red window in figure 3. The maximum pixel value within ORI is represented as $Z_{t o p}$, while the minimum one is represented as $Z_{b o t}$. Their corresponding relative irradiance are $L_{t o p}$ and $L_{b o t}$, respectively. The dynamic range of the ORI is referred as optical dynamic range (ODR), represented as $\eta$. It can be got through formula (2). ODR is a key parameter showing the ability of a camera to capture high dynamic range images. ODR differs for various kinds of cameras. The over-exposing and low-exposing can be avoided if the whole dynamic range is divided into several parts and each part is mapped within ORI. In this way, a complete information of weld pool can be obtained by the camera.

This can be realized by the irradiance-based imaging algorithm proposed in this paper. It takes images of the welding scene by changing exposure time. The exposure time can be represented as a set $\left\{\Delta t_{1}, \ldots, \Delta t_{N}\right\}$. Every exposure time $\Delta t_{k}$ from the set is used to capture one image respectively. The order will be from $\Delta t_{1}$ to $\Delta t_{N}$, then loop. $k \in[1, N]$, and $N$ is the number of the exposure times. The set of exposure time $\left\{\Delta t_{1}, \ldots, \Delta t_{N}\right\}$ is decided in advance based on the welding scene irradiance. It starts by finding the first exposure time $\Delta t_{1}$ :

(a) Setting the exposure time $\Delta T^{(k)}=\Delta t_{\min } . \Delta t_{\min }$ is the minimum exposure time that the camera can set. At first, $k=1$.

(b) Take an image with $\Delta T^{(k)}$ as the exposure time, and traverse the brightness $Z(i, j)$ of all the image pixels.

(c) If every $Z(i, j) \notin(0,255), \Delta T^{(k+1)}=10 * \Delta T^{(k)}$. Repeat step (b) as iteration.

(d) If exist a pixel $Z(i, j) \in(0,255)$, stop the iteration and output the image $I_{0}$ captured with the final $\Delta T^{(k)}$, as shown in figure $4(\mathrm{a})$.

(e) Binarize $I_{0}$ with the Otsu method[18] and get a logical image, as shown in figure 4(b). The area with a logic value of 1 is represented as $R_{c}$, shown in white in the image, corresponding to the area with the highest brightness in the original welding image.

(f) Find the geometric center $p_{o}$ of $R_{c}$, and its brightness $Z_{p 0}$. Then get the relative irradiance $L_{p 0}$ according to formula (1).

(g) By letting $p_{o}$ have a middle pixel value $Z_{\text {mid }}=128, \Delta t_{1}$ can determined by:

$$
\Delta t_{1}=\exp \left(g\left(Z_{\text {mid }}\right)\right) / \mathrm{A} L_{p 0}
$$

The determination of the rest exposure time in $\left\{\Delta t_{1}, \ldots, \Delta t_{N}\right\}$ obeys the following rules. As mentioned above, the exposure time $\left\{\Delta t_{1}, \ldots, \Delta t_{N}\right\}$ are used to capture images and every image records a part of the welding information within the ORI of the camera, as shown in figure 5. As a result, every image has its own range of relative irradiance $L^{k}$ which covers a certain region of the whole welding scene irradiance. If these regions are combined together by overlapping with each other, then the summed up dynamic range can be as wide as that of the welding scene. The overlapping law satisfies that the bottom limit of the first region is set to be equal to the top limit of the next region: 


$$
L_{b o t}^{k}=L_{t o p}^{k+1}
$$

Where $L_{b o t}^{k}$ is the minimum irradiance of the ORI of the $k^{\text {th }}$ image while $L_{t o p}^{k+1}$ is the maximum irradiance of the ORI of the $(k+1)^{\text {th }}$ image. Therefore, every image is captured within the ORI of the camera and avoid the use of the range out of ORI.

For the $k^{\text {th }}$ image and the $(k+1)^{t h}$ image, there exists:

$$
\left\{\begin{array}{c}
f\left(Z_{b o t}, \Delta t_{k}, L_{b o t}^{k}\right)=0 \\
f\left(Z_{\text {top }}, \Delta t_{k+1}, L_{\text {top }}^{k}\right)=0
\end{array}\right.
$$

By combining formula (4) and (5), the relationship is established between two adjacent exposure times, $\Delta t_{k}$ and $\Delta t_{k+1}$ :

$$
\frac{\Delta t_{k+1}}{\Delta t_{k}}=\exp \left(g\left(Z_{\text {top }}\right)-g\left(Z_{\text {bot }}\right)\right)
$$

By applying formula (3) to formula (6), $\left\{\Delta t_{1}, \ldots, \Delta t_{K}\right\}$ can be obtained, as shown in figure 6. Because $\Delta t_{1}$ makes sure that the brightest area in the image is well-exposed and $\Delta t_{1}<\Delta t_{2} \ldots<\Delta t_{K}$, the whole region avoids over-exposed. By using this method, the camera can be adaptive to the dynamic range change of the welding scene, avoiding the imaging problem countered with AGC strategy.

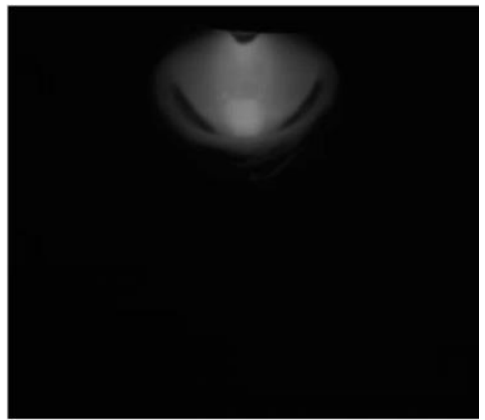

(a)

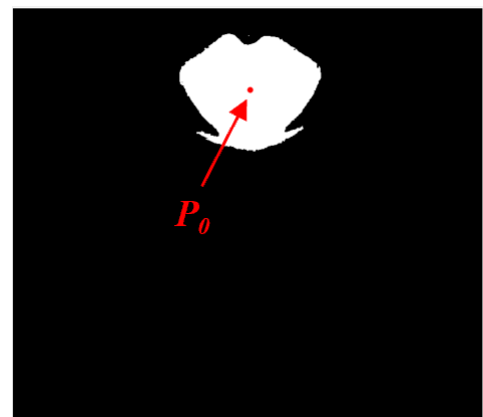

(b)

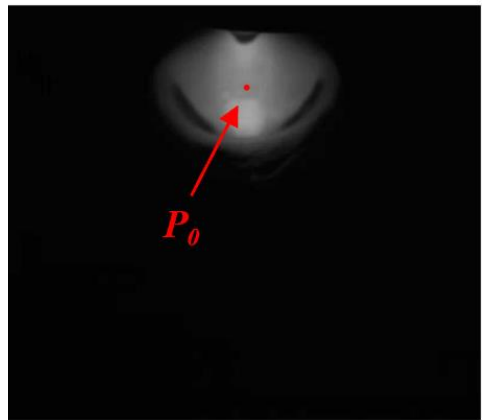

(c)

Fig. 4 The determination of the first exposure time $\Delta t_{1}$. (a)first frame capture by the camera. (b)center point $p_{o}$ extraction from the binary image of (a). (c) Letting brightness of $p_{o}$ to be 128 when capturing image with $\Delta t_{1}$.

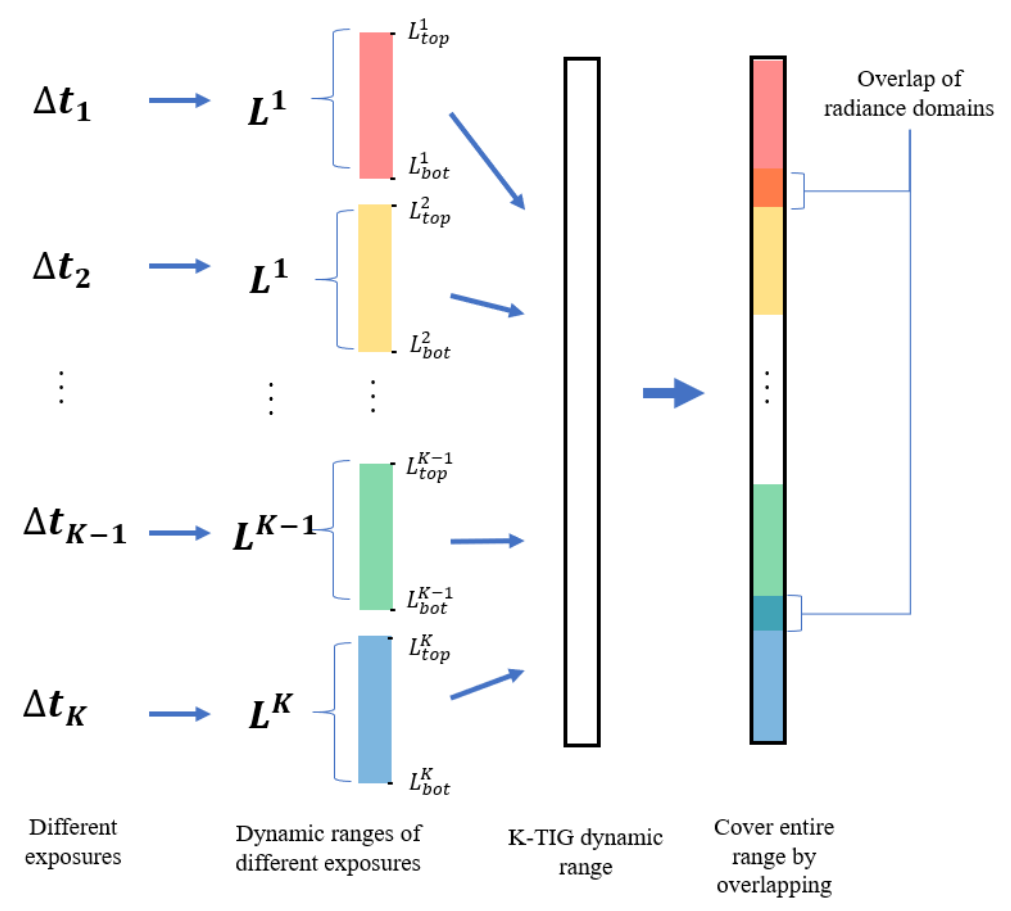

Fig. 5 The strategy of widening dynamic range of welding image. 


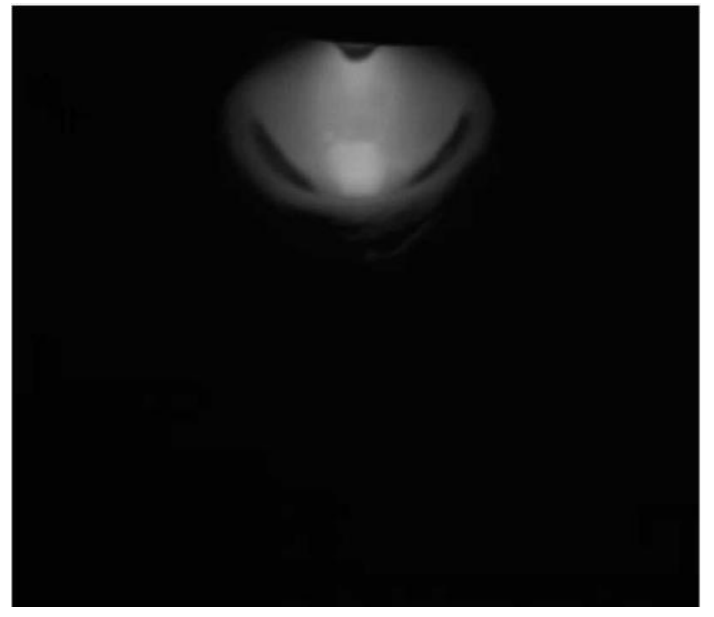

(a)

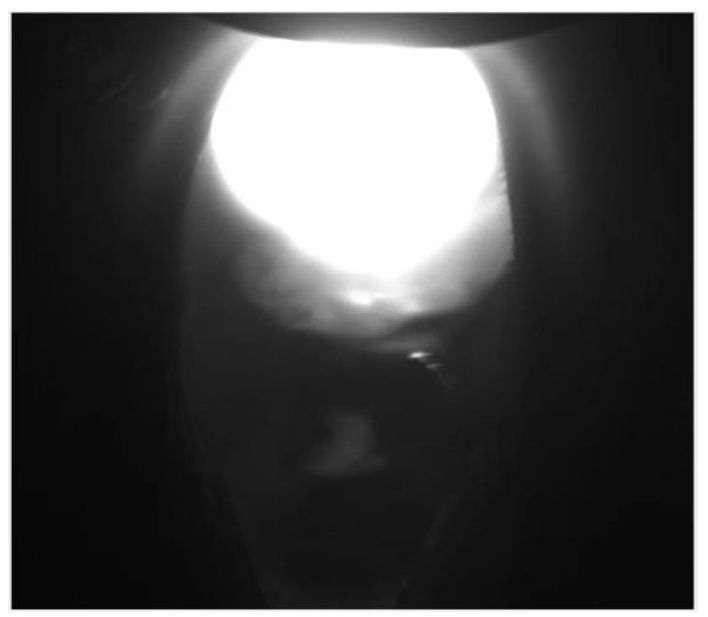

(c)

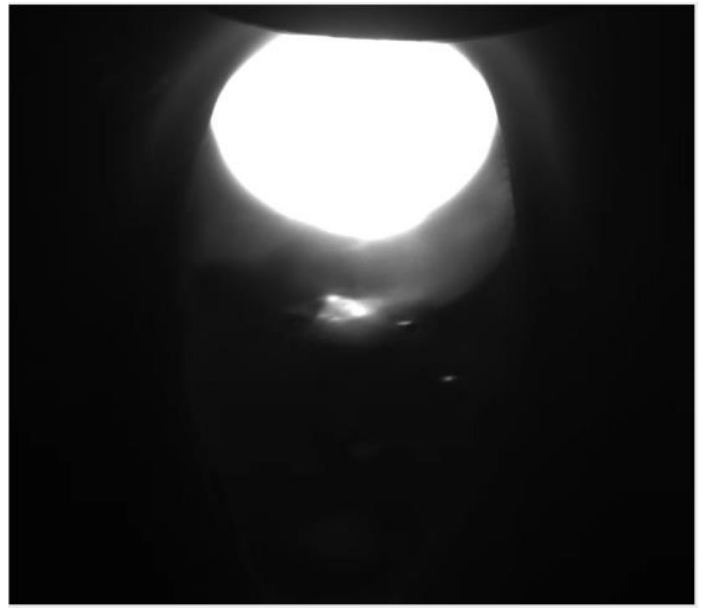

(b)

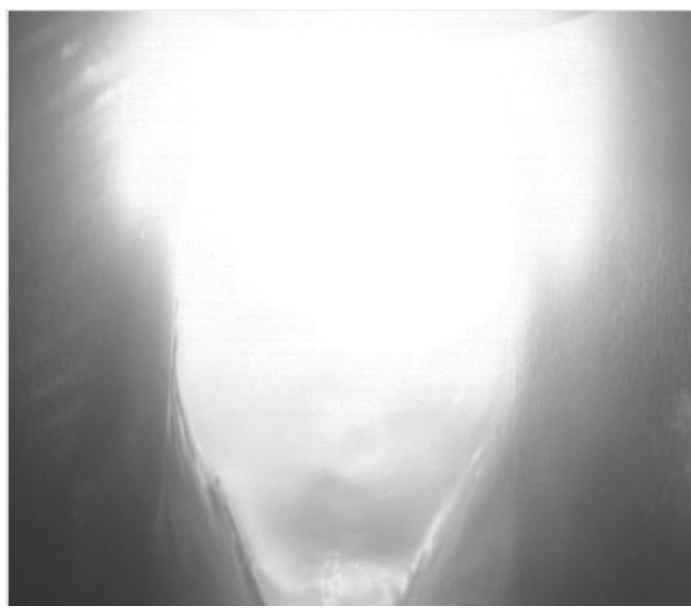

(d)

Fig. 6 The welding images capture with the set $\left\{\Delta t_{1}, \ldots, \Delta t_{K}\right\}$ where $K=4$. (a) $\Delta t_{1}=40 \mu \mathrm{s}$. (b) $\Delta t_{2}=5000 \mu \mathrm{s}$. (c) $\Delta t_{3}=12800 \mu \mathrm{s}$. (d) $\Delta t_{4}=100000 \mu \mathrm{s}$. 


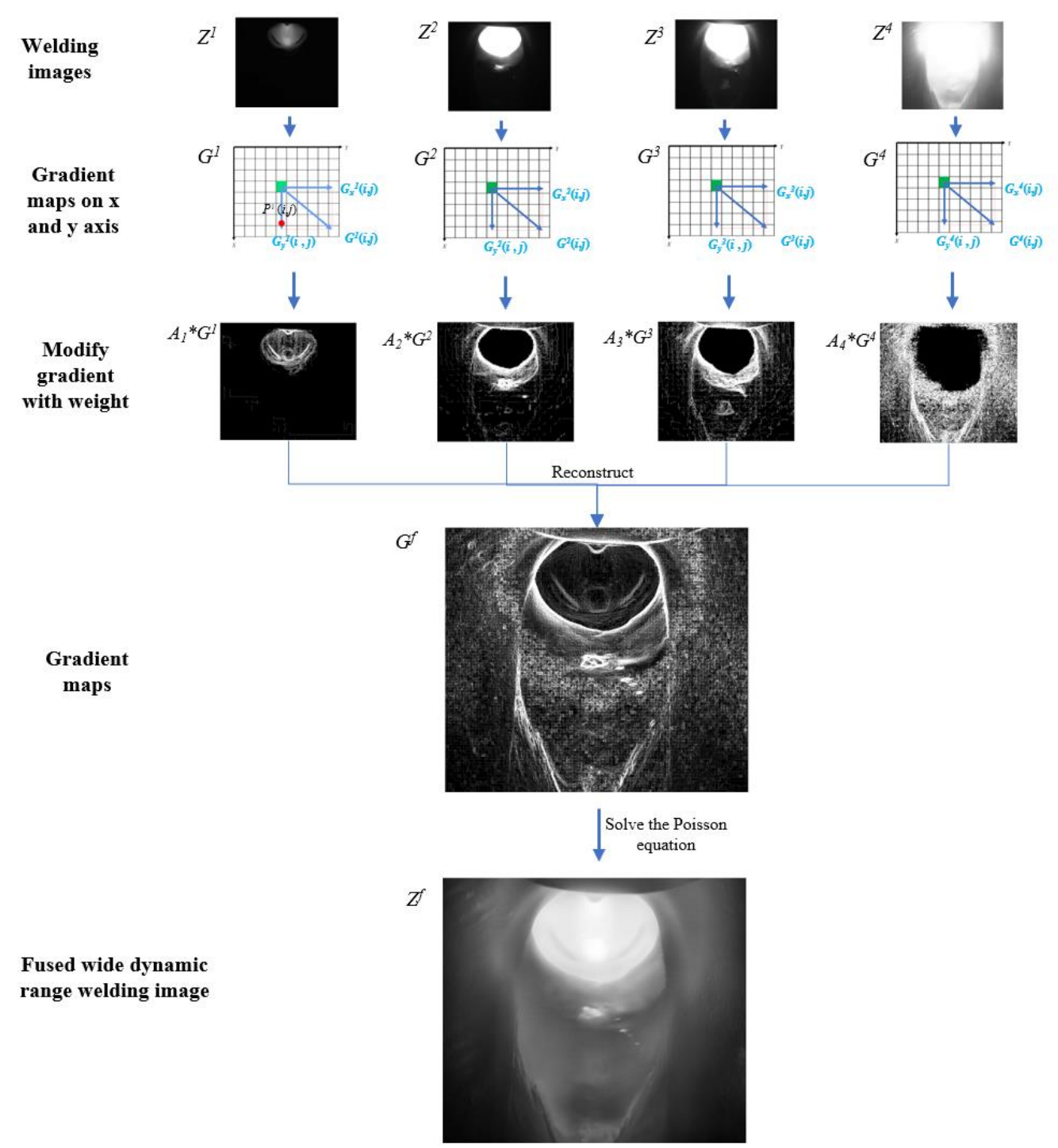

Fig. 7 The process of obtaining the high-dynamic-range welding image by image fusion algorithm

\subsection{Welding image fusion}

Although a wider dynamic range is obtained by taking a set of images with irradiance-based imaging algorithm, the features of the weld pool seem discontinued from the images. It means parts of a feature was shown in different brightness in different image. It brings difficulties in welding monitoring as well as the feature automatic detection. In this part, an image fusion method is used to reconstructed a high-dynamic-range image, avoiding disconnected and unnatural artifacts. The process of the image fusion is shown in figure 7. For the $k^{\text {th }}$ image captured by irradiance-based imaging algorithm, the pixel value at the position of $(i, j)$ is referred to $Z(i, j) . i$ is the $x$ axis coordinate and $j$ is $y$ axis coordinate in the image coordinate. First, the gradient map of the image is obtained by:

$$
\boldsymbol{G}^{\boldsymbol{k}}(i, j)=\boldsymbol{G}_{\boldsymbol{x}}^{\boldsymbol{k}}(i, j)+\boldsymbol{G}_{\boldsymbol{y}}^{\boldsymbol{k}}(i, j)
$$

Where $\boldsymbol{G}^{\boldsymbol{k}}(i, j)$ represents the total gradient vector at the position of $(i, j) . \boldsymbol{G}_{\boldsymbol{x}}^{\boldsymbol{k}}(i, j)$ and $\boldsymbol{G}_{\boldsymbol{y}}^{\boldsymbol{k}}(i, j)$ are the gradient vectors in the direction of $x$ axis and $y$ axis respectively. Because the pixel value matrix can be seen as a discrete domain, the modulus of $\boldsymbol{G}_{\boldsymbol{x}}^{\boldsymbol{k}}(i, j)$ and $\boldsymbol{G}_{\boldsymbol{y}}^{\boldsymbol{k}}(i, j)$ can be given by:

$$
\left\{\begin{array}{l}
\left|\boldsymbol{G}_{\boldsymbol{x}}^{\boldsymbol{k}}(i, j)\right|=|Z(i+1, j)-Z(i, j)| \\
\left|\boldsymbol{G}_{\boldsymbol{y}}^{\boldsymbol{k}}(i, j)\right|=|Z(i, j+1)-Z(i, j)|
\end{array}\right.
$$


Every $G^{k}(i, j)$ shows the information of the edges in the images, so the higher it is the more features is around this pixel. Therefore, at every pixel $(i, j)$, the maximum $\boldsymbol{G}^{\boldsymbol{k}}(i, j)$ is selected from $k$ images and referred as $\boldsymbol{G}^{f}(i, j)$ :

$$
\boldsymbol{G}^{\boldsymbol{f}}(i, j)=A_{k} * \max _{1 \leq k \leq N}\left\{\left|\boldsymbol{G}^{\boldsymbol{k}}(i, j)\right|\right\}
$$

Where $A_{k}$ is the weight corresponding to the index of image. It makes the pixels with higher brightness have a greater weight while reconstructing the final gradient map $\boldsymbol{G}^{f}$. In this study, $A_{1}=1.2, A_{2}=1.1, A_{3}=1.0, A_{4}=1.0 . G^{f}$ becomes a new gradient map which contains the maximum feature information. It can be seen as the gradient map of a new image $Z^{f}$, which is the pixel value of the fused image. Then the function between the new gradient and the pixel value at every point of the welding scene is described as:

$$
G^{f}=\nabla Z^{f}
$$

Where $\nabla$ represents the $\left\{\frac{d}{d i}, \frac{d}{d j}\right\}$. In order to solve for $Z^{f}$, the formula (10) is changed into a form of Poisson equation:

$$
\nabla^{T} G^{f}=\nabla^{2} Z^{f}
$$

It aims to avoid the calculation problem caused by the unconservative of the field of gradient[19]. In this paper, a method based on the Haar wavelets[20] is used which relates the Haar wavelet decomposition coefficients of $Z^{f}$ and the fused gradient $G^{f}$. Finally, an iteration form to get the fused image can be described as[21]:

$$
Z_{n}^{f}=Z_{(n-1)}^{f}+0.25\left(M_{1} \otimes Z_{y(n-1)}^{f}+M_{2} \otimes G_{x(n-1)}^{f}-M_{3} \otimes Z_{(n-1)}^{f}\right)
$$

Where $n$ is the number of the iteration and $\otimes$ is the convolution operator. $M_{1}, M_{2}$ and $M_{3}$ are the constant matrixes:

$$
M_{1}=\left[\begin{array}{ccc}
-1 & 0 & -1 \\
0 & 4 & 0 \\
-1 & 0 & -1
\end{array}\right], M_{2}=\left[\begin{array}{cc}
1 & -1 \\
1 & -1
\end{array}\right], M_{3}=\left[\begin{array}{cc}
1 & 1 \\
-1 & -1
\end{array}\right]
$$

By setting a factor, the iteration will stop when pixel value mean of the fused image is closed to this factor. The lowest image in the figure 7 is the final image got by image fusion. It contains more information of the weld pool, including the internal feature like keyhole and the external profile of the pool.

\subsection{Operating window for optimal image fusion}

The adaptive image fusion can greatly increase the dynamic range of the images captured by the camera. However, the performance of the algorithm depends on two factors. One is the ODR of the camera. According to section 3.1, the final dynamic range of the new image is a multiple of ODR, which means the higher ODR of the camera, the better performance of the algorithm will have. The other one is the minimum exposure time $\Delta t_{\text {min }}$ that the camera can set. During the image capturing, the total time cost for a fused time is equal to the sum of all the exposure times. The next expose time is several times of the former one. Therefore, the value of the $\Delta t_{\min }$ will affect the fluency and image quality of the video stream. Experiments and derivation are carried out in this section to determine the operating window for optimal image fusion. Assuming that $\Delta t_{1}=t_{\min }$, so that the total time cost will be as low as possible. Let $\eta$ represents the value of ODR. The $\Delta t_{k}$ and the total time cost $T_{\text {total }}$ can be described as:

$$
\begin{aligned}
& \Delta t_{k}=\eta \Delta t_{k-1}=\eta^{k} t_{\text {min }} \\
& T_{\text {total }}=\sum_{k=1}^{K} \Delta t_{k}=\frac{t_{0}\left(\eta^{K}-1\right)}{\eta-1}
\end{aligned}
$$


Where $K$ is the number of images used for fusion. In order to ensure the fluency of the video stream, there is a frequency threshold $f_{v}$, otherwise the video will be choppy:

$$
\frac{t_{\min }\left(\eta^{K}-1\right)}{\eta-1} \leq \frac{1}{f_{v}}
$$

In this study, $f_{v}$ is set to be $10 \mathrm{~Hz}$ and the camera has a $\eta$ of $2.6 \mathrm{~dB}$ and a $t_{\min }$ of $38 \mu \mathrm{s}$. By adding the number of images used for image fusion to up to its limit $\left(K_{\max }=4\right)$, the total dynamic range of the fused image increases linearly and reaches its maximum, as shown as the third curve in figure 8(a). In order to capture the keyhole TIG welding process, the dynamic range of fused image has to higher than that of welding scene $(8.3 \mathrm{~dB})$. Therefore, the law of choosing the propriate number of images is $K=K_{\max }$.

According to Formula (15), the changes of $t_{\min }$ and $\eta$ will have impact on the total dynamic range and $K_{\max }$. Experiments were taken to find out an operating window for the setting of these two factors. When the $t_{\min }$ is set to be constant, $\eta$ is adjusted to see the changes, as shown in figure 8(a). As the $\eta$ increases, the slope of the curve increases as well, making it easier to get a wider dynamic range with a smaller number of images. But if the $\eta$ is too large or too small, the total dynamic range decreases. As a result, $\eta$ should be chosen within a range. When the $t_{\min }$ is changed, the maximum dynamic range of the fused image changes as well, as shown in figure 8(b). On one hand, if the If the $t_{\min }$ is too large, it is impossible to capture keyhole TIG welding because the curve is under the welding scene line. On the other hand, if the $t_{\min }$ is small enough, the area under the curve and above the welding scene line will be appropriate for the optimal parameter configuration. In conclusion, the number of the images used for image fusion should be as many as possible to widen the dynamic range. Besides, the rise of $\eta$ can increase the $K_{\max }$, but too large $\eta$ will do harm to the dynamic range widening. There is an optimal choice of $\eta$ which maximizes the dynamic range. Finally, the choice of the $t_{\min }$ should be small in order to get a high dynamic range.

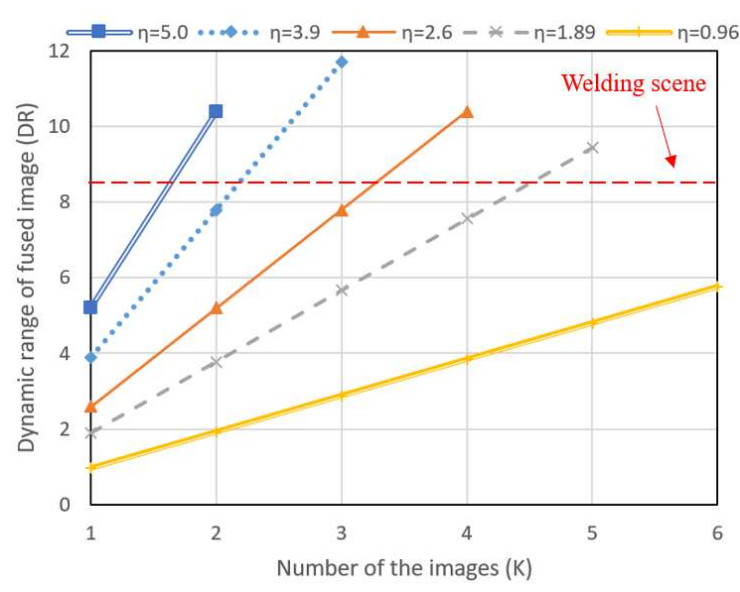

(a)

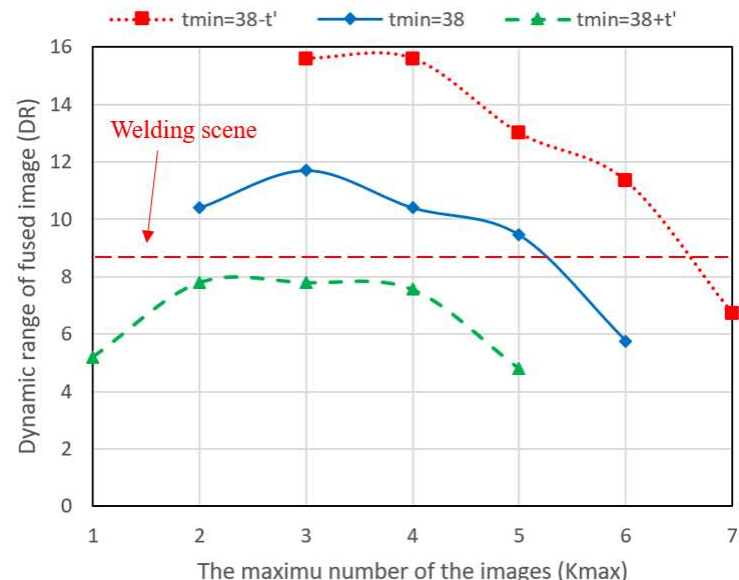

(b)

Fig. 8 Operating windows for the optimal image fusion. (a) The impact of $\eta$ on dynamic range of fussed image (b) The impact of $t_{\min }$ on dynamic range of fussed image.

\section{Internal and external feature detection of the weld pool}

The detection of the features from the welding pool is usually uncomplete because of the narrow-dynamic range of the monitoring system. Once a part of the features is obtained, the other parts will be badly exposed in the image. It causes the lack of the information when the welding monitoring is carried out, resulting in errors in features detection. Therefore, the simultaneous detection of both the internal and external feature is valuable. It enables the geometry extraction of the whole weld pool instead of only a portion of it. A complete detection of features of the weld pool can be realized based on the images obtained by the adaptive image fusion algorithm. In this section, the width of the keyhole entry is 
detected and the edge fitting of the weld pool head and its tail is also realized. It does help to the complete information detection of the weld pool during the keyhole TIG welding, solving the difficulty in the internal monitoring, as shown in figure 9.

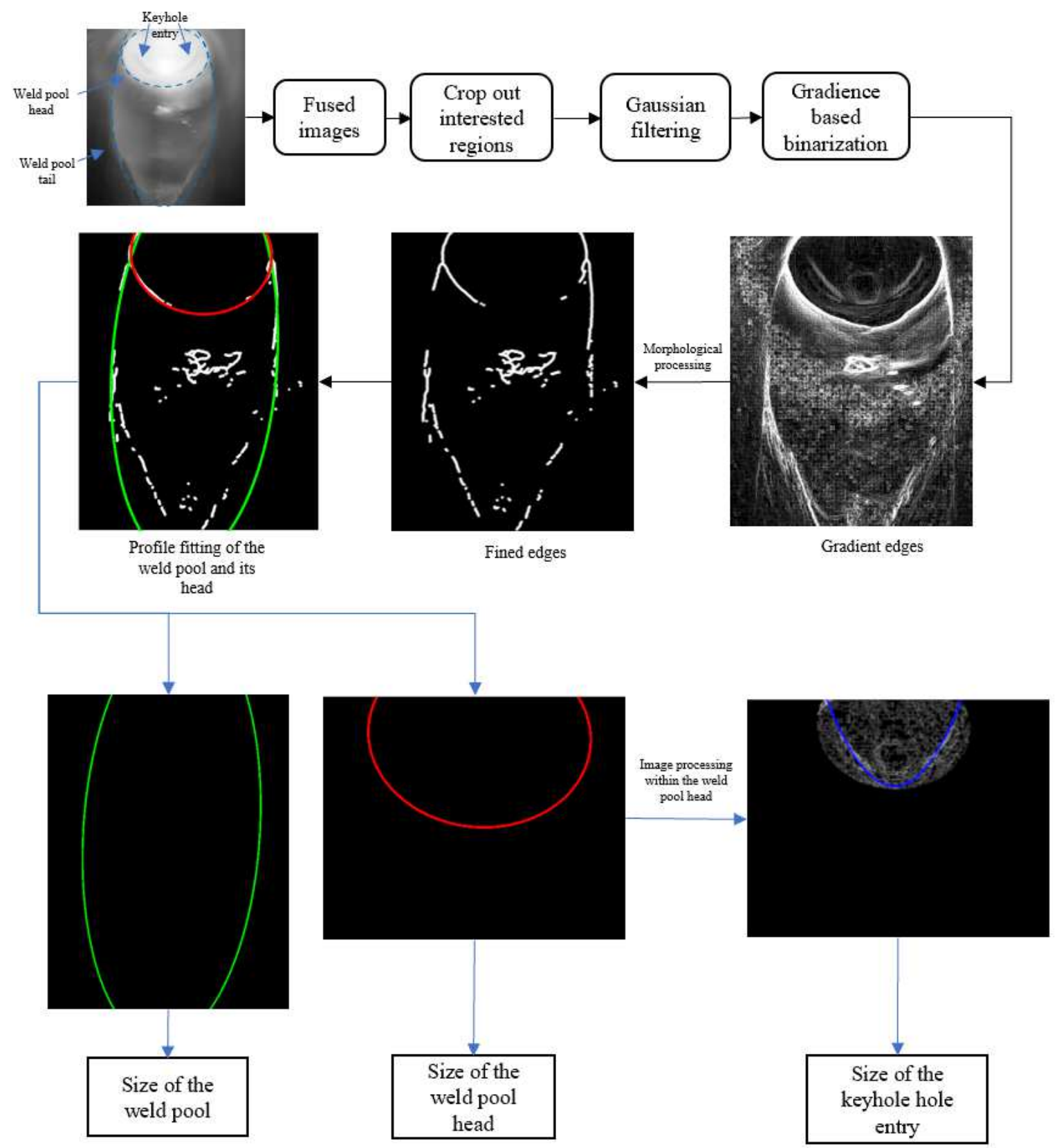

Fig. 9 Extraction of the internal and external features of the weld pool based on adaptive image fusion.

The first step is to detect the profile of the whole weld pool. By applying the Sobel operator[22], the image is turned into gradient field. Then the interested region is cropped out before applying binarization based on statistic of the gradience value. Geometric processing is used to delete the isolated region and preserve the useful edge. Finally, the edges are refined to get the edges. The second step is to detect three pairs of typical point for the pool head fitting. The first pair of points is the two pixels which the edges intersects with the top boundary of the image. The second pair of points is chosen as the interaction of the edge of the head of pool and the tail of pool. The third pair of points is on the edge of the tail of pool, and the horizonal distance between these two reaches its maximum. The first and second pairs of points are used to detect the boundary of the head of pool, while the first and third pairs of points are used to tail of 
pool. After that, the boundary of the head and tail of pool is fitted. The third step is the detection of the keyhole entry by applying image processing algorithm within the head of pool. After crop out the region of weld pool head from the gradient map, the edge extraction and curve fitting can be realized. After the process mentioned above, the key features from the weld pool is completely extracted, including the profile of the whole weld pool, outline of the weld pool head and the size of the keyhole entry. These can be realized through an image on a single visual sensor, improving the accuracy and efficiency of the detection of the weld pool features.

\section{Results and analysis}

Comparison of the imaging quality is made on the CMOS camera between those with and without adaptive image fusion. Experiments are carried out twice with the same welding parameters, which ensures the weld pool geometry can be assumed to be the same. The camera is used to capture images of keyhole TIG welding process. One is with the proposed algorithm and the other is without. The distribution of the brightness of the pixels in both images are analyzed in figure 10. The image without algorithm records the welding pool with less information. A lot of pixels exceed its brightness value (reaching 255) inside the weld pool head. In addition, the weld pool tail keeps low brightness, resulting in difficulty in the weld pool tail edge recognition, as shown in figure 10(a). By comparison, the image with fusion algorithm shows more diversity of the brightness, having more different value within the weld pool head while the weld pool tail remains a high level of brightness, which helps to detection the edges, as shown in figure 10(b). In order to further discuss the performance of the proposed algorithm, three lines are chosen from the image. They are $0 \mathrm{~mm}, 15 \mathrm{~mm}$ and $26 \mathrm{~mm}$ away from the position of the torch tip. All the pixels on these three lines correspond to three different positions on the weld pool. Because the camera is calibrated for its position, the same position of lines can be selected from the image from two experiments with or without proposed image fusion algorithm, as shown in figure 11(a)(b). The pixel values on these three lines are extracted to see the changes of pixel value along the row. For the line under the torch tip, it goes through the region of the keyhole inside the weld pool, as shown in figure 11(c). The pixel value of the reaches the maximum pixel value in the middle of the curve in the original image, while the step change on the curve from the fused image shows the details from the keyhole. For the line of $15 \mathrm{~mm}$ away from the torch tip, both two curve shows irregular ups and downs in pixel value, as shown in figure 11(d). It could be owed to the reflection from the arc light one the weld pool surface. For the line of $26 \mathrm{~mm}$ from the torch tip, as shown in figure 11(e). It goes through the tail of the weld pool. The curve in original image is relatively flat and hardly to show the edge of weld pool tail. By contrast, the curve in fused image has two peaks in the middle, corresponding to the left and right boundaries of the weld pool tail. To sum up, the image captured with the adaptive image fusion can be adaptive to the brightness of the keyhole region, avoiding the over-exposure of the pixel in the keyhole area. Meanwhile, the boundary of the weld pool tail can also be recorded clearly. In addition, the edges information is reserved which does help to the feature extraction in the monitoring process.

The results of the size measurement of the features in weld pool region are analyzed below. The width of the weld pool and the width of the weld pool head are detected. In addition, due to the use of the adaptive image fusion, the keyhole inside the weld pool can be extracted. The highest coefficient of the fitted parabola is considered to be the curvature of the keyhole entry geometry. The results are shown in figure 12. At the beginning of the welding process, the arc pressure is applied to the weldment but the penetration is not realized. Therefore, the heat input is transferred to the weldment, causing a large amount of metal melted and form the weld pool. As a result, the width of the whole weld pool and its head 
becomes wider. As the welding process becomes stable, the keyhole is formed and the torch travels for a certain distance. A portion of the input heat is leaking from the keyhole exit at the bottom and transferred to the other part of the weldment. Therefore, the width of the weld pool and becomes smaller and reaches a stable value which is close to the width of the weld pool head. During the whole process, the arc pressure digs out a cavity in the liquid metal no matter whether the keyhole is formed or not, so the curvature changes due to the oscillation of the molten metal but remains within a certain range. The result shows that the complete information from the weld pool can be extraction via the adaptive image fusion, such as the internal and external features of the weld pool. Besides, the accurate data can be obtained by the measurement of the width or curvature, which have potential to reflect the welding process information like quality. The proposed method can be a new way to analysis the process of the keyhole TIG welding.

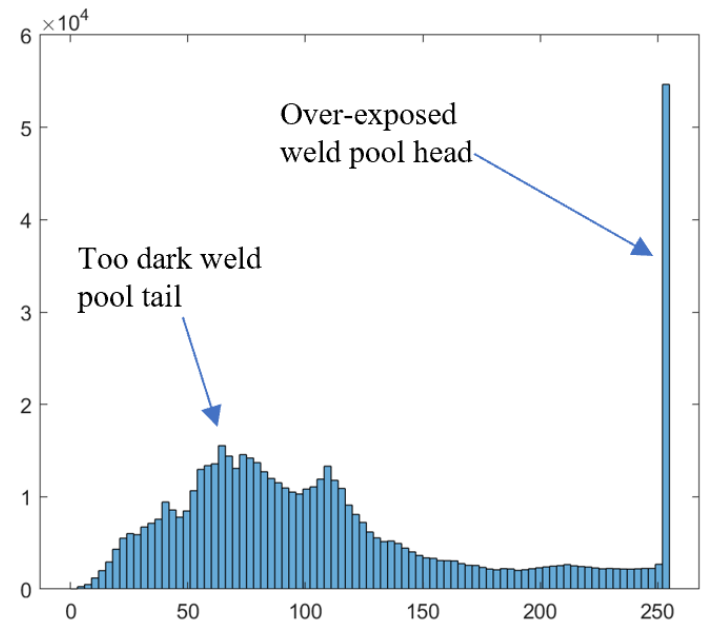

(a)

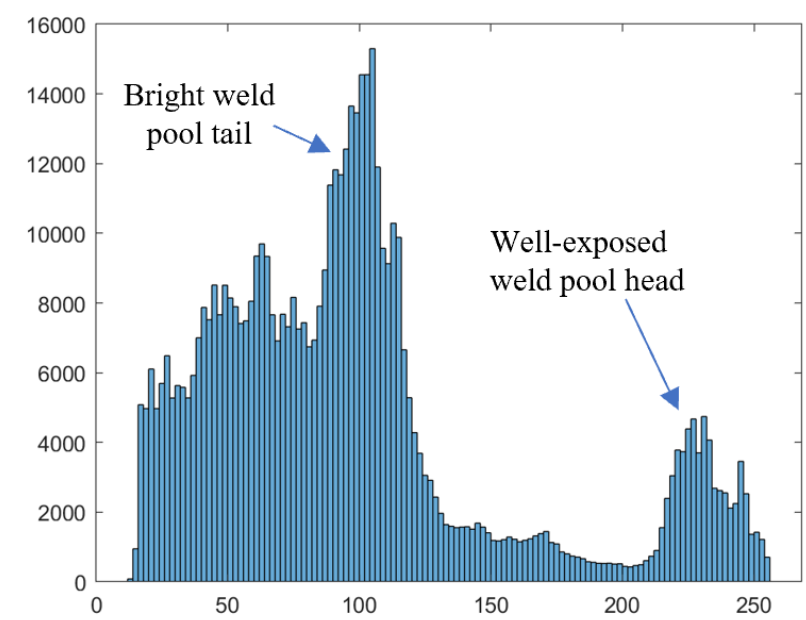

(b)

Fig. 10 The comparison of the image pixel brightness histogram. (a)image without the algorithm, (b) image with the algorithm.

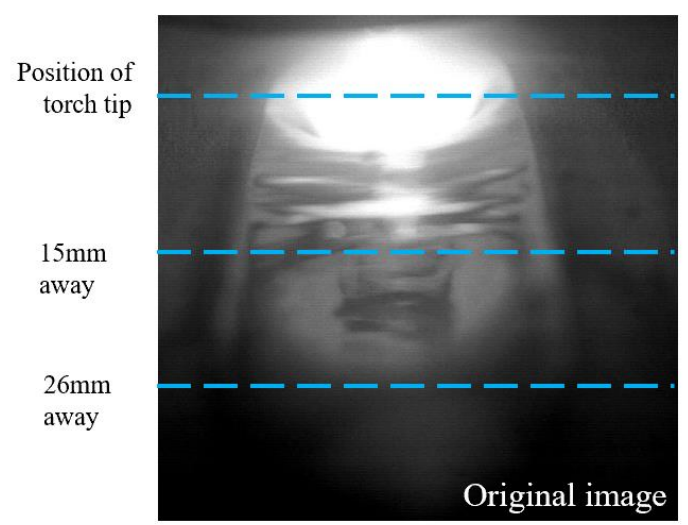

(a)

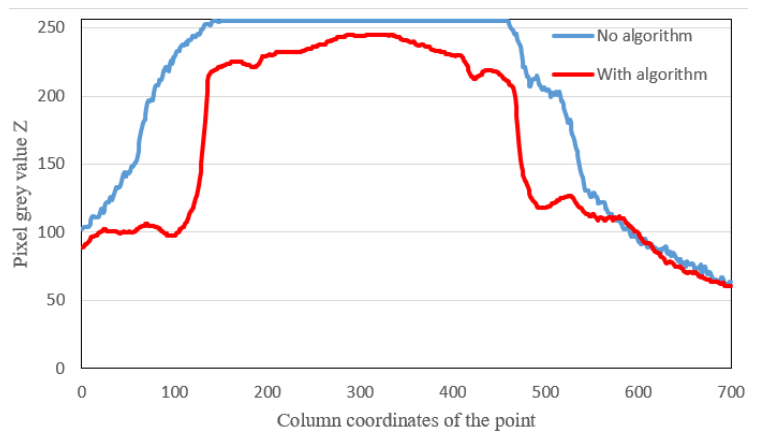

(c)

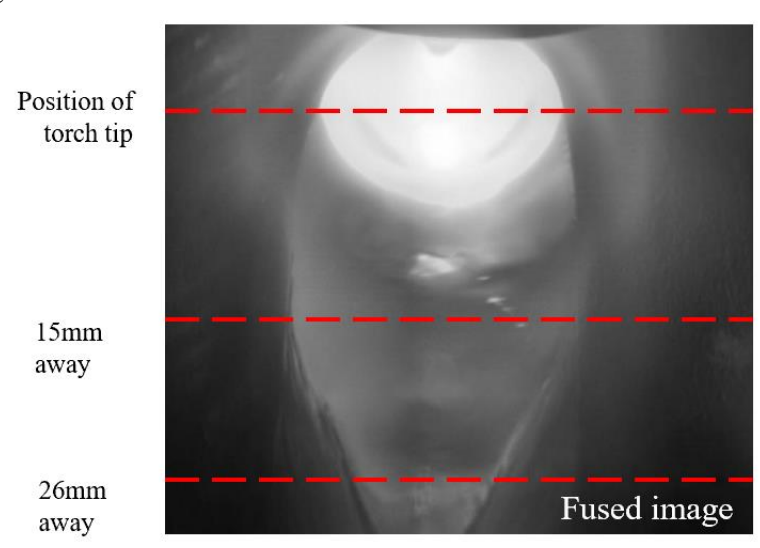

(b)

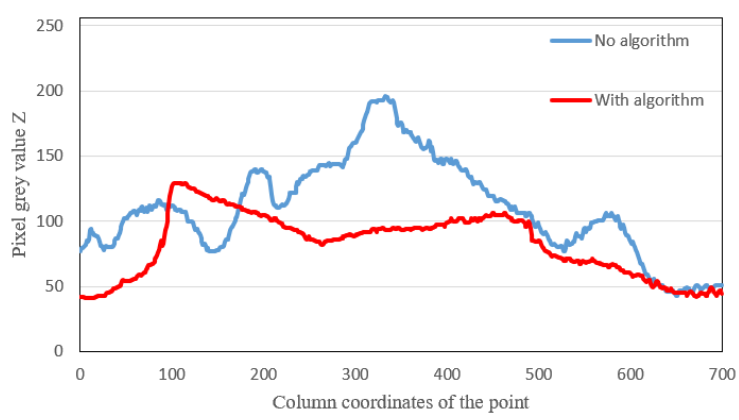

(d) 


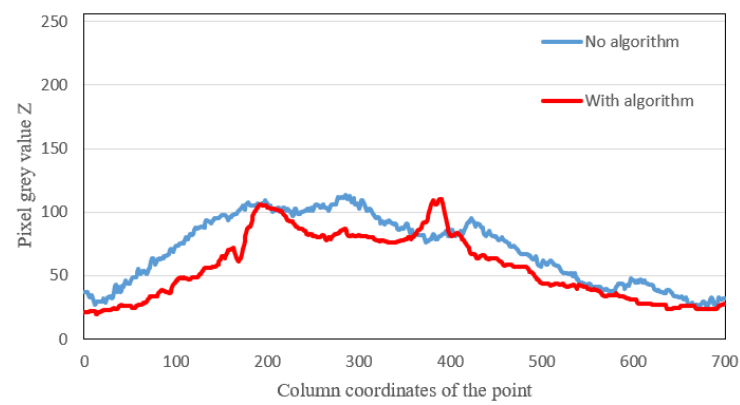

(e)

Fig. 11 The improvement of the image with the proposed algorithm by comparison. (a)image without the algorithm, (b) image with the algorithm, (b) pixel values on the line below the torch tip, (d) pixel values on the line $15 \mathrm{~mm}$ away from the torch tip, (e) pixel values on the line $26 \mathrm{~mm}$ away from the torch tip.

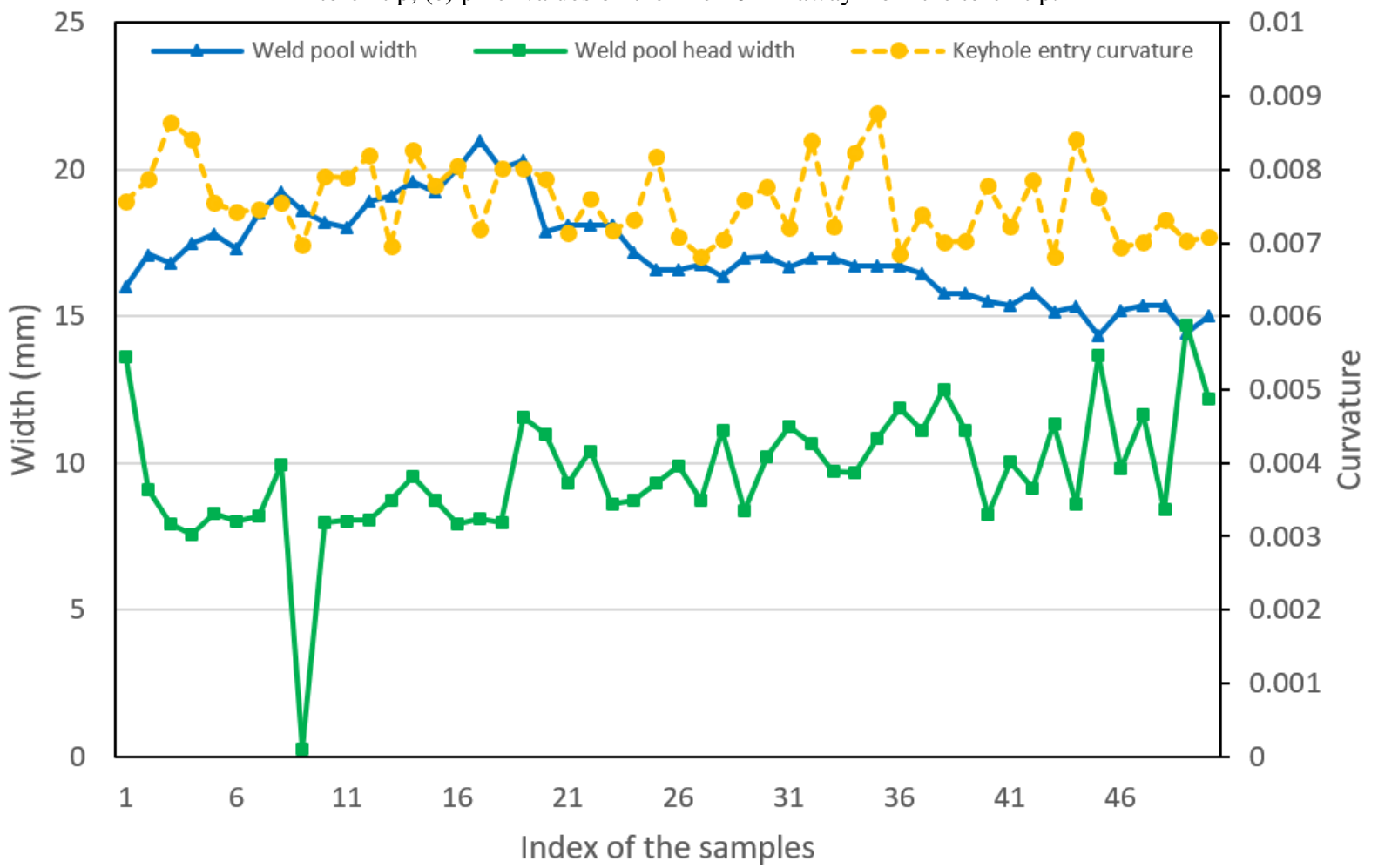

Fig. 12 Changes of the widths of the weld pool and its head and the curvature of the keyhole entry.

\section{Conclusion}

The high dynamic range of the keyhole TIG welding scene is measured by experiments. It is found to be the reason for bringing difficulties to the clear monitoring of the weld pool region as well as the detection of the internal features of the weld pool. In this paper, an adaptive image fusion algorithm is proposed to obtain a new image of the weld scene. It can not only extend the dynamic range of the welding images, but can also has the potential to keep good imaging quality when fusing the images captured under different welding parameters. Based on this, the extraction of the features of keyhole and the weld pool is realized. The main contributions of this study are concluded as follows:

1. The reason for the visual monitoring problem encountered in keyhole TIG welding is found to be the dynamic range of the scene is greater than the dynamic range of the camera.

2. An adaptive image fusion algorithm is proposed to widen the dynamic range of the welding image and ensure that the weld pool with high contrast can be well exposed when the welding situation changes.

3. The operation window for adaptive image fusion algorithm is got, including the choice of the 
optical range for imaging and the minimum exposure time of the camera.

4. The simultaneous feature extraction of the whole welding zone is realized, including the internal and external regions of the weld pool.

\section{Funding}

This study is financially supported from the Key Research and Development Program of Guangdong Province (Grant No. 2020B090928003), and the Natural Science Foundation of Guangdong Province (Grant No. 2020A1515011050).

\section{Conflicts of interest/Competing interests}

The authors declared that they do not have any commercial or associative interest that represents a conflict of interest in connection with the work submitted.

\section{Availability of data and material}

Not applicable

\section{Code availability}

Not applicable

\section{Authors' contributions}

(1) The dynamic range of the keyhole welding scene was measured, which is considered to the reason for over-exposing in camera monitoring.

(2) A high-dynamic-range visual sensing method was proposed to solve the over-exposing problem caused by the strong arc light.

(3)Achieve the simultaneous extraction of the features of both weld pool and keyhole of K-TIG welding.

(4) The optimal operating window for proposed sensing algorithm was found for industrial application.

\section{Ethics approval}

All procedures performed in studies involving human participants were in accordance with the ethical standards of the institutional and/or national research committee

\section{Consent to participate}

I believe the participant is giving informed consent to participate in this study

\section{Consent for publication}

I would like to declare on behalf of my co-authors that the work described was original research that has not been published previously, and not under consideration for publication elsewhere, in whole or in part. All the authors listed have approved the manuscript that is enclosed. 


\section{References}

1. Y. Shi, S. Cui, T. Zhu, S. Gu, X. Shen, Microstructure and intergranular corrosion behavior of HAZ in DP-TIG welded DSS joints, Journal of Materials Processing Technology 256 (2018) 254-261.

Doi: 10.1016/j.jmatprotec.2018.02.019.

2. Zhang BR, Shi YH, Gu SY. Narrow-seam identification and deviation detection in keyhole deep-penetration TIG welding. INT J ADV MANUF TECH 2019;101:5-8.

Doi : 10.1007/s00170-018-3089-0

3. Jarvis, B.L., and N.U. Ahmed. (2000) Development of Keyhole Mode Gas Tungsten Arc Welding Process. Science and Technology of Welding and Joining, 5, (1), pp. 21-1718.

$10.1179 / 136217100322910624$

4. Xia, C., Pan, Z., Fei, Z., Zhang, S. and Li, H. (2020). Vision based defects detection for Keyhole TIG welding using deep learning with visual explanation. Journal of Manufacturing Processes, 56, pp.845-855.

Doi: 10.1016/j.jmapro.2020.05.033

5. Wang, X., Shi, Y., Yu, G., Liang, B. and Li, Y. (2016). Groove-center detection in gas metal arc welding using a template-matching method. The International Journal of Advanced Manufacturing Technology, 86(9-12), pp.2791-2801.

Doi: 10.1007/s00170-016-8389-7

6. Bakir, N., Pavlov, V., Zavjalov, S., Volvenko, S., Gumenyuk, A. and Rethmeier, M. (2018). Novel metrology to determine the critical strain conditions required for solidification cracking during laser welding of thin sheets. Journal of Physics: Conference Series, 1109, p.012047.

Doi :10.1088/1742-6596/1109/1/012047

7. De, A., and D. Parle. "Real Time Seam Tracking System for Automated Fusion Arc Welding." Science and Technology of Welding and Joining, vol. 8, no. 5, Oct. 2003, pp. 340-346, 10.1179/136217103225005471. Accessed 1 June 2020

8. Luo, M. and Shin, Y.C. (2015). Vision-based weld pool boundary extraction and width measurement during keyhole fiber laser welding. Optics and Lasers in Engineering, 64, pp.59-70.

Doi : 10.1016/j.optlaseng.2014.07.004

9. Shao, Wen Jun, et al. (2018) A Novel Weld Seam Detection Method for Space Weld Seam of Narrow Butt Joint in Laser Welding. Optics \& Laser Technology, 99, pp. 39-51.

10.1016/j.optlastec.2017.09.037.

10. Fang, J. and Wang, K. (2019). Weld Pool Image Segmentation of Hump Formation Based on Fuzzy C-Means and Chan-Vese Model. Journal of Materials Engineering and Performance, 28(7), pp.4467-4476.

Doi : 10.1007/s11665-019-04168-y

11. Tang, D., Wang, K., Duan, R., Niu, H. and Long, B. (2019). Research on Quality Control of Arc Welding Robot Based on Molten Pool Contour extraction. IOP Conference Series: Materials Science and Engineering, 490, p.052001.

Doi : 10.1088/1757-899x/490/5/052001

12. Jiang, C., Zhang, F. and Wang, Z. (2017). Image Processing of Aluminum Alloy Weld Pool for Robotic VPPAW Based on Visual Sensing. IEEE Access, 5, pp.21567-21573.

Doi : 10.1109/access.2017.2761986

13. Cui, Y., Shi, Y. and Hong, X. (2019). Analysis of the frequency features of arc voltage and its application to the recognition of welding penetration in K-TIG welding. Journal of Manufacturing Processes, 46, pp.225-233.

Doi : 10.1016/j.jmapro.2019.09.002

14. Debevec, Paul E. and Malik, Jitendra (2008) Recovering High Dynamic Range Radiance Maps from Photographs. Association for Computing Machinery, 30(11).

$10.1145 / 1401132.1401174$ 
15. Banterle, F., Debattista, K., Artusi, A., Pattanaik, S., Myszkowski, K., Ledda, P. and Chalmers, A. (2009). High Dynamic Range Imaging and Low Dynamic Range Expansion for Generating HDR Content. Computer Graphics Forum, 28(8), pp.2343-2367.

$10.1111 / \mathrm{j} .1467-8659.2009 .01541 . x$

16. El Gamal, A. and Eltoukhy, H. (2005). CMOS image sensors. IEEE Circuits and Devices Magazine, 21(3), pp.6-20.

Doi : $10.1109 / \mathrm{mcd} .2005 .1438751$

17. Cho, J. (2010). A Real-Time Histogram Equalization System with Automatic Gain Control Using FPGA. KSII Transactions on Internet and Information Systems.

Doi : $10.3837 /$ tiis.2010.08.011

18. Otsu, N., 1979. A Threshold Selection Method from Gray-Level Histograms. IEEE Transactions on Systems, Man, and Cybernetics, 9(1), pp.62-66.

19. Wrobel, Bernard P. (2021). Least-Squares Methods for Surface Reconstruction from Images. ISPRS Journal of Photogrammetry and Remote Sensing, vol. 46, no. 2, Apr. 1991, pp. 67-84.

Doi : 10.1016/0924-2716(91)90017-p

20. Hampton, P.J., Agathoklis, P. and Bradley, C. (2008). A New Wave-Front Reconstruction Method for Adaptive Optics Systems Using Wavelets. IEEE Journal of Selected Topics in Signal Processing, 2(5), pp.781-792.

Doi : 10.1109 /jstsp.2008.2006386

21. Paul, S., Sevcenco, I.S. and Agathoklis, P. (2016). Multi-Exposure and Multi-Focus Image Fusion in Gradient Domain. Journal of Circuits, Systems and Computers, 25(10), p.1650123.

Doi : $10.1142 / \mathrm{s} 0218126616501231$

22. N. Kanopoulos, N. Vasanthavada and R. L. Baker (1988) Design of an image edge detection filter using the Sobel operator, in IEEE Journal of Solid-State Circuits, 23(2), pp. 358-367.

10.1109/4.996. 


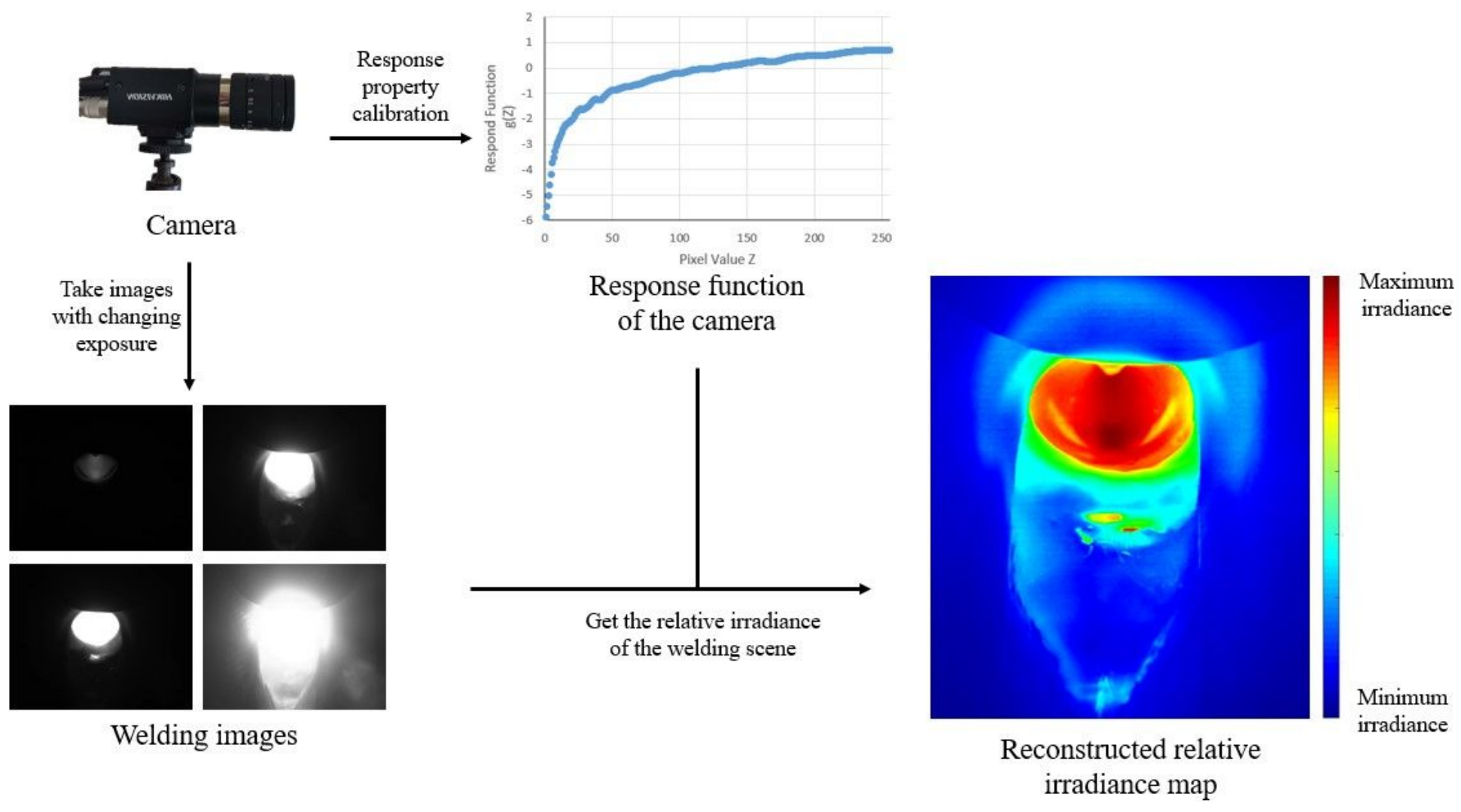

Figure 1

The process of the measurement of the dynamic range of the welding scene 


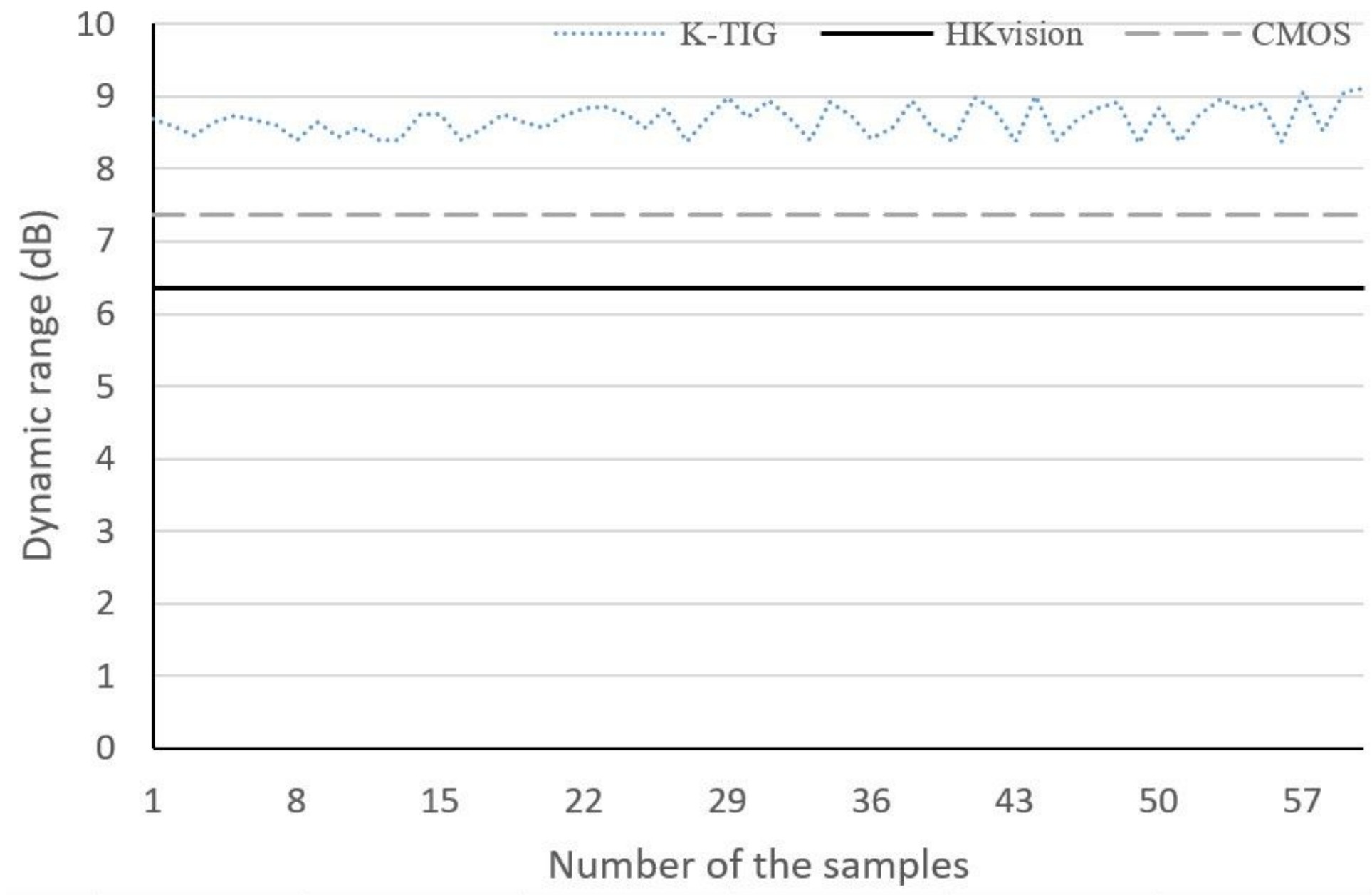

Figure 2

The comparison of the dynamic range of the welding scene and camera

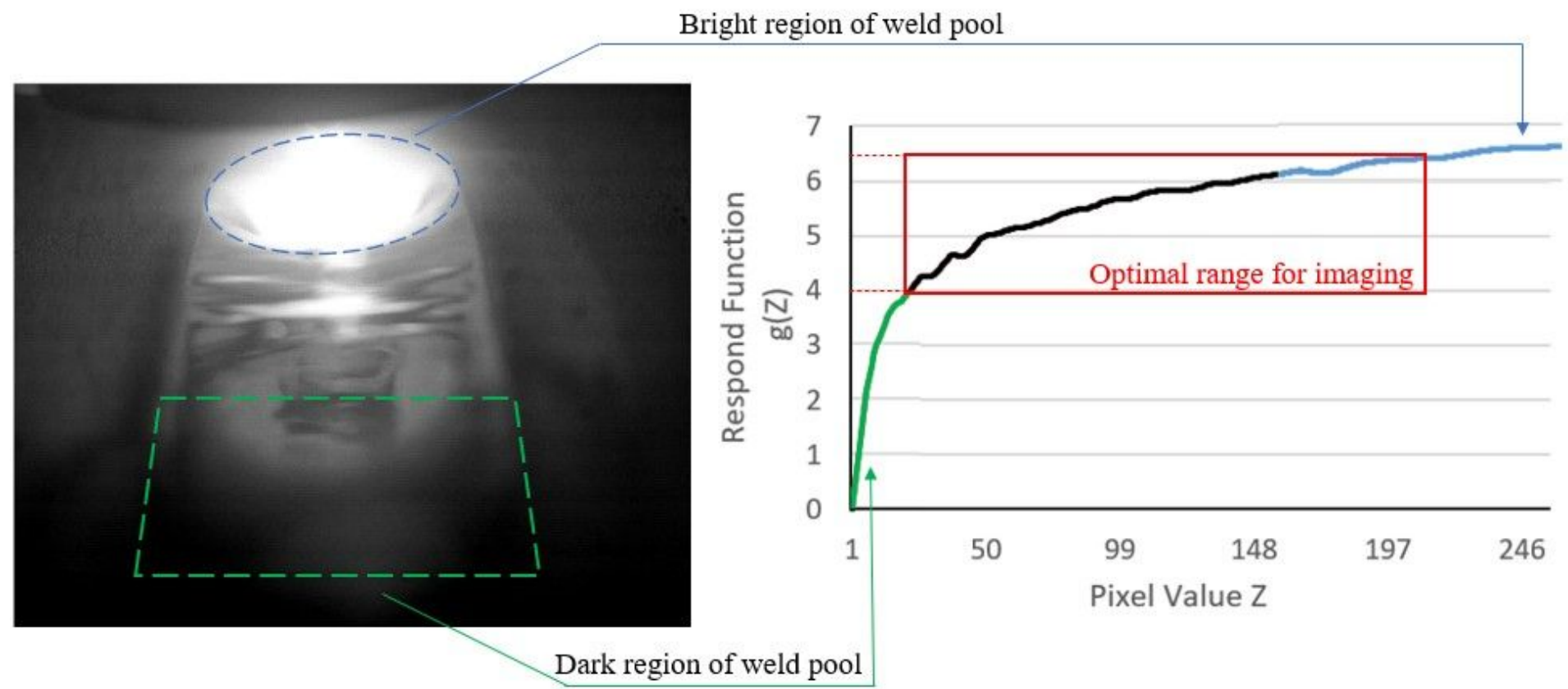

Figure 3 
Analysis of the different portion of the response function curve of the camera.

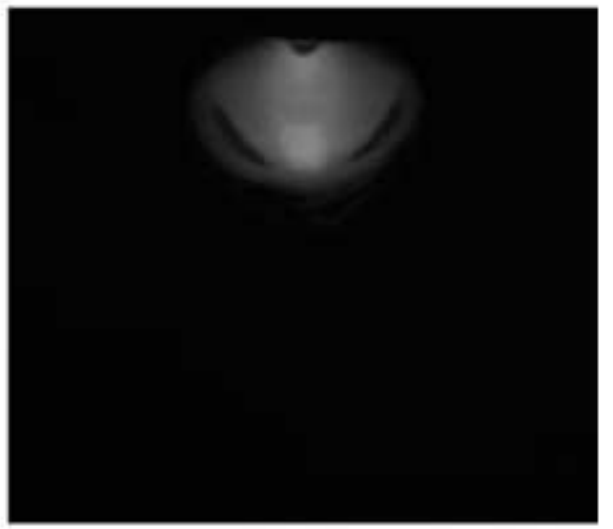

(a)

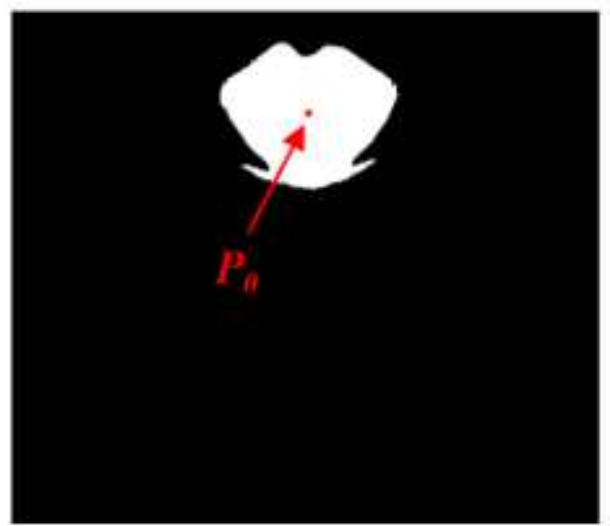

(b)

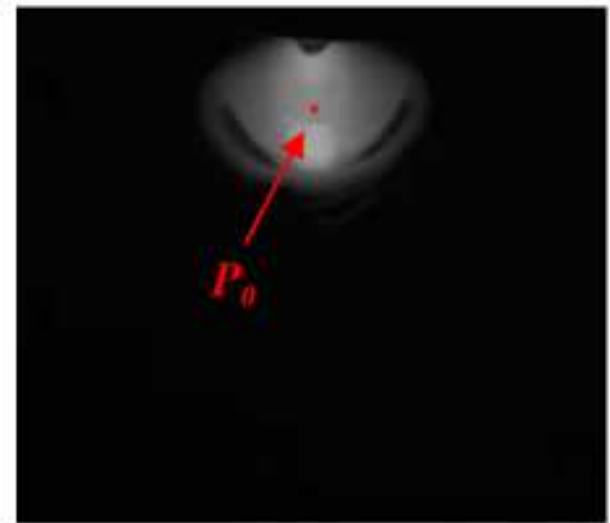

(c)

Figure 4

See manuscript for full figure caption. 


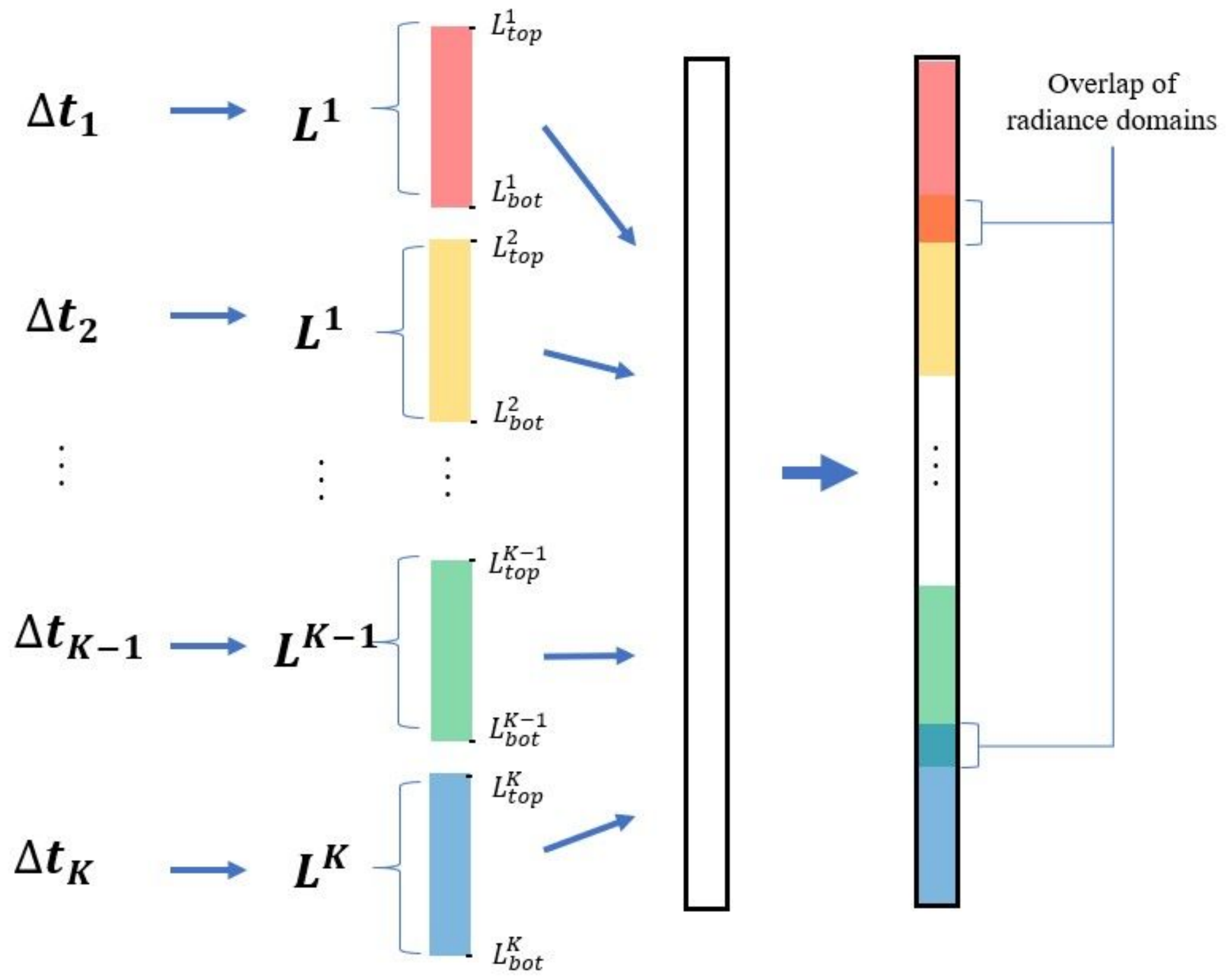
Different
Dynamic ranges of
exposures
different exposures
K-TIG dynamic range
Cover entire
range by
overlapping

Figure 5

The strategy of widening dynamic range of welding image. 


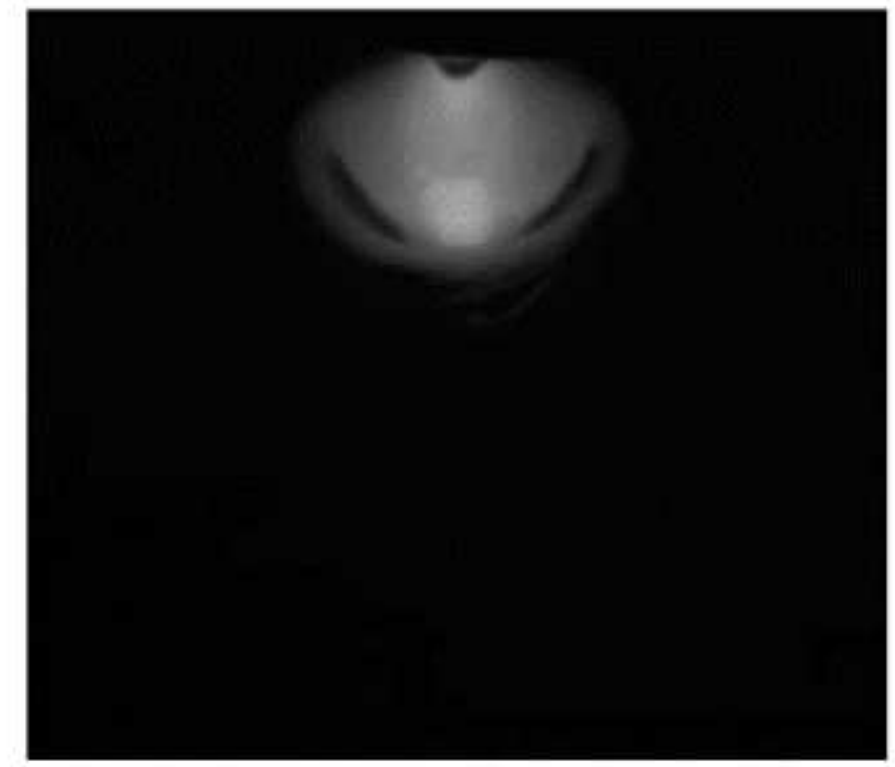

(a)

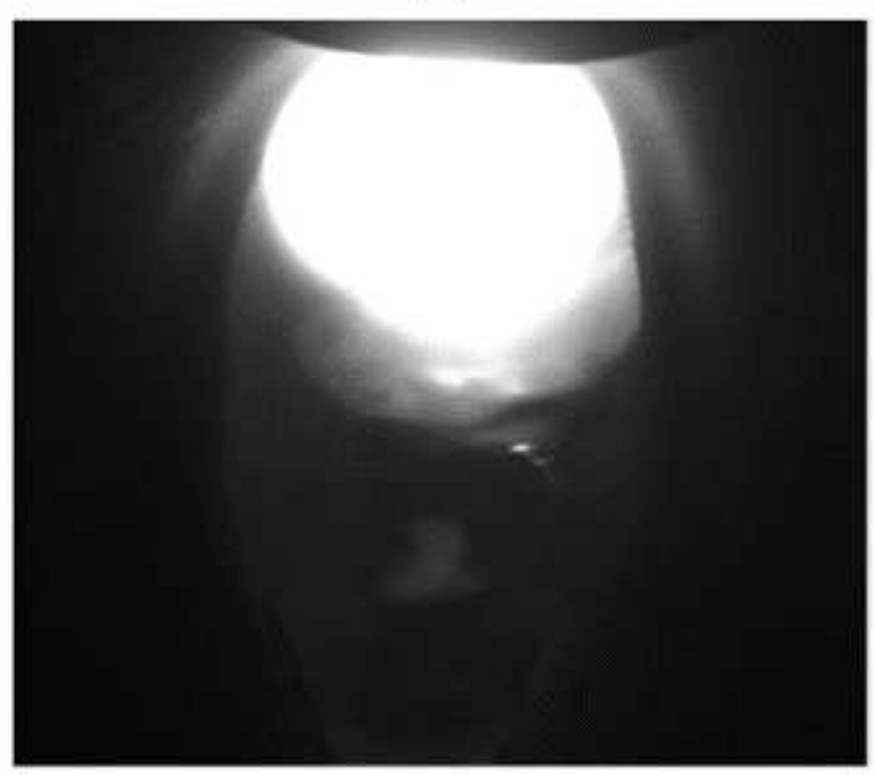

(c)

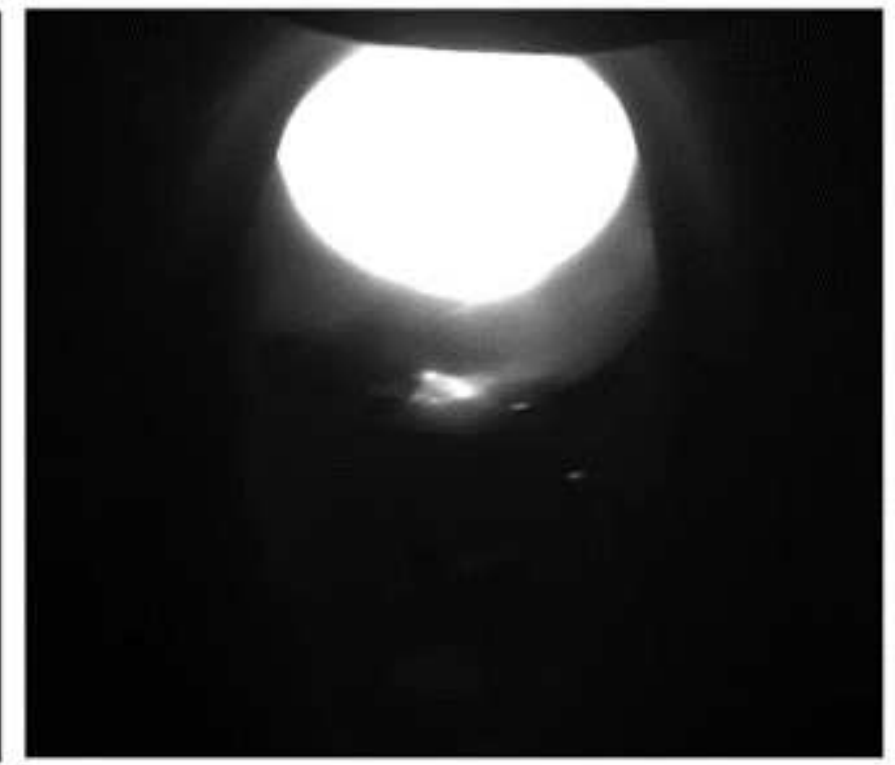

(b)

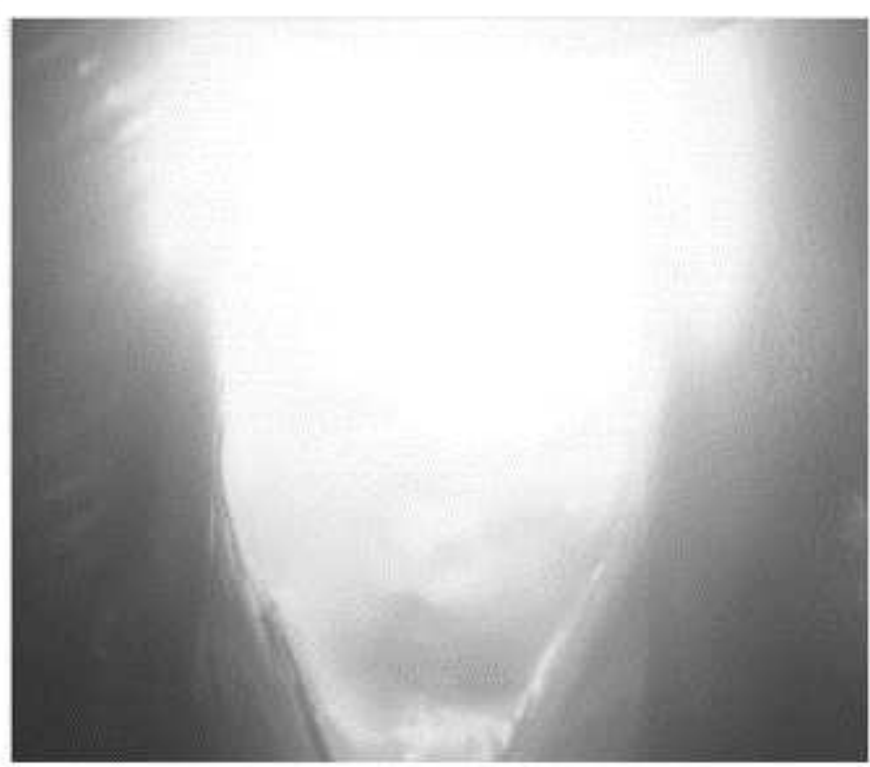

(d)

Figure 6

See manuscript for full figure caption. 


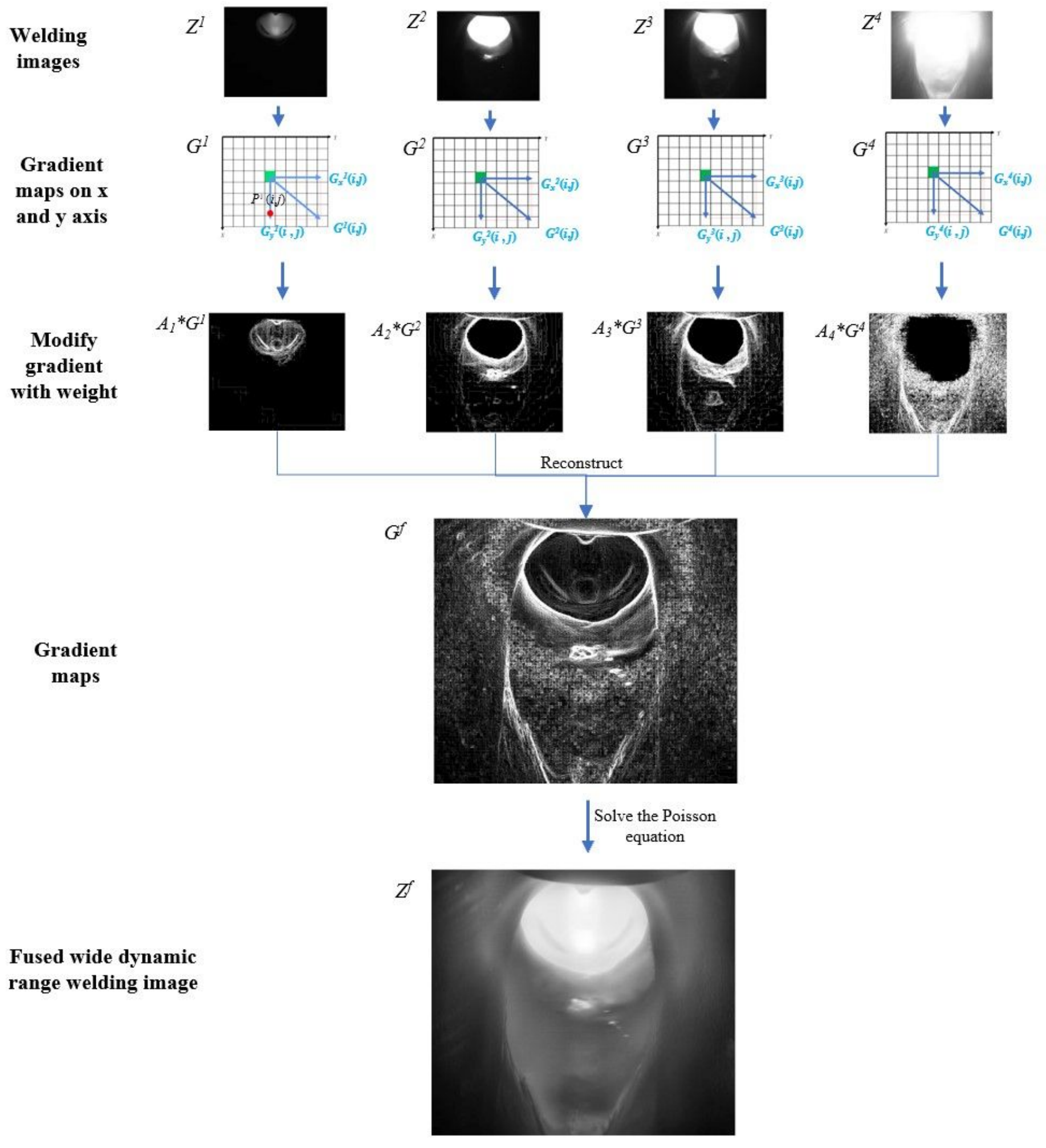

Figure 7

The process of obtaining the high-dynamic-range welding image by image fusion algorithm 


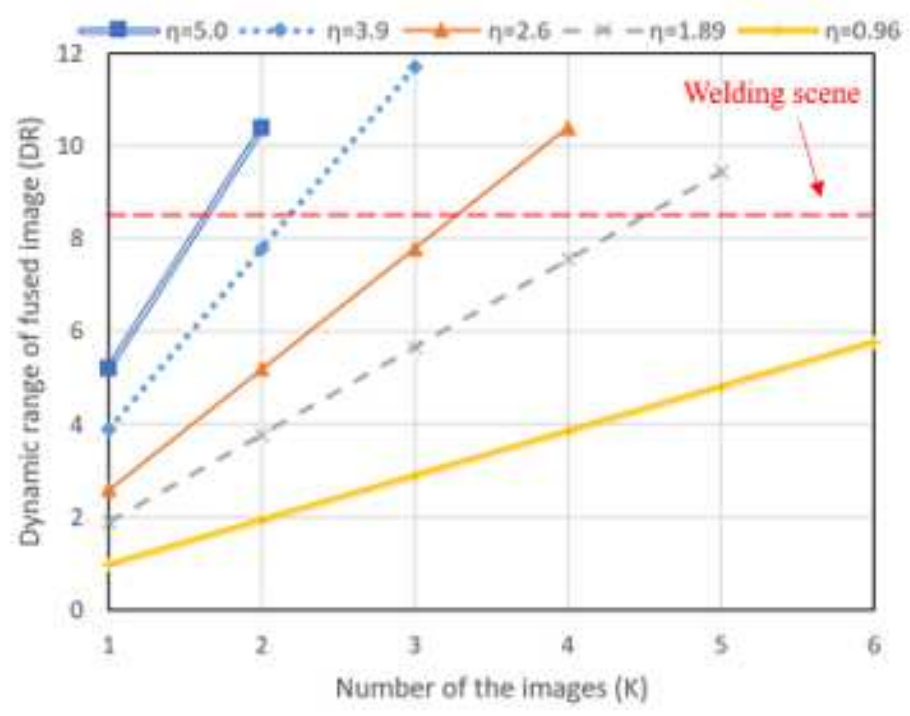

(a)

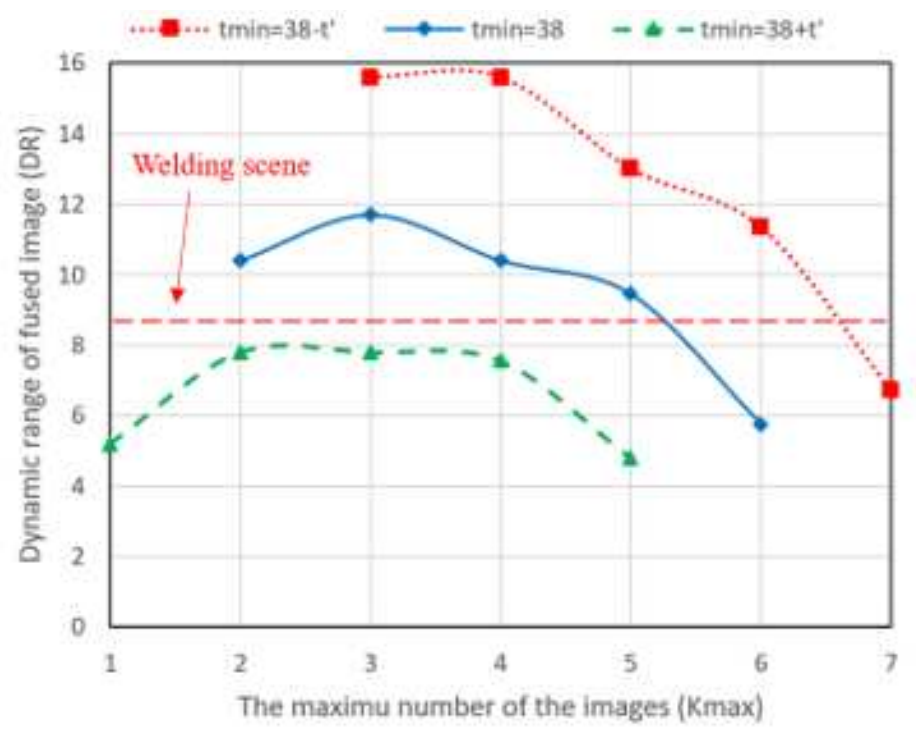

(b)

\section{Figure 8}

Operating windows for the optimal image fusion. (a) The impact of $\eta$ on dynamic range of fussed image (b) The impact of t_min on dynamic range of fussed image. 


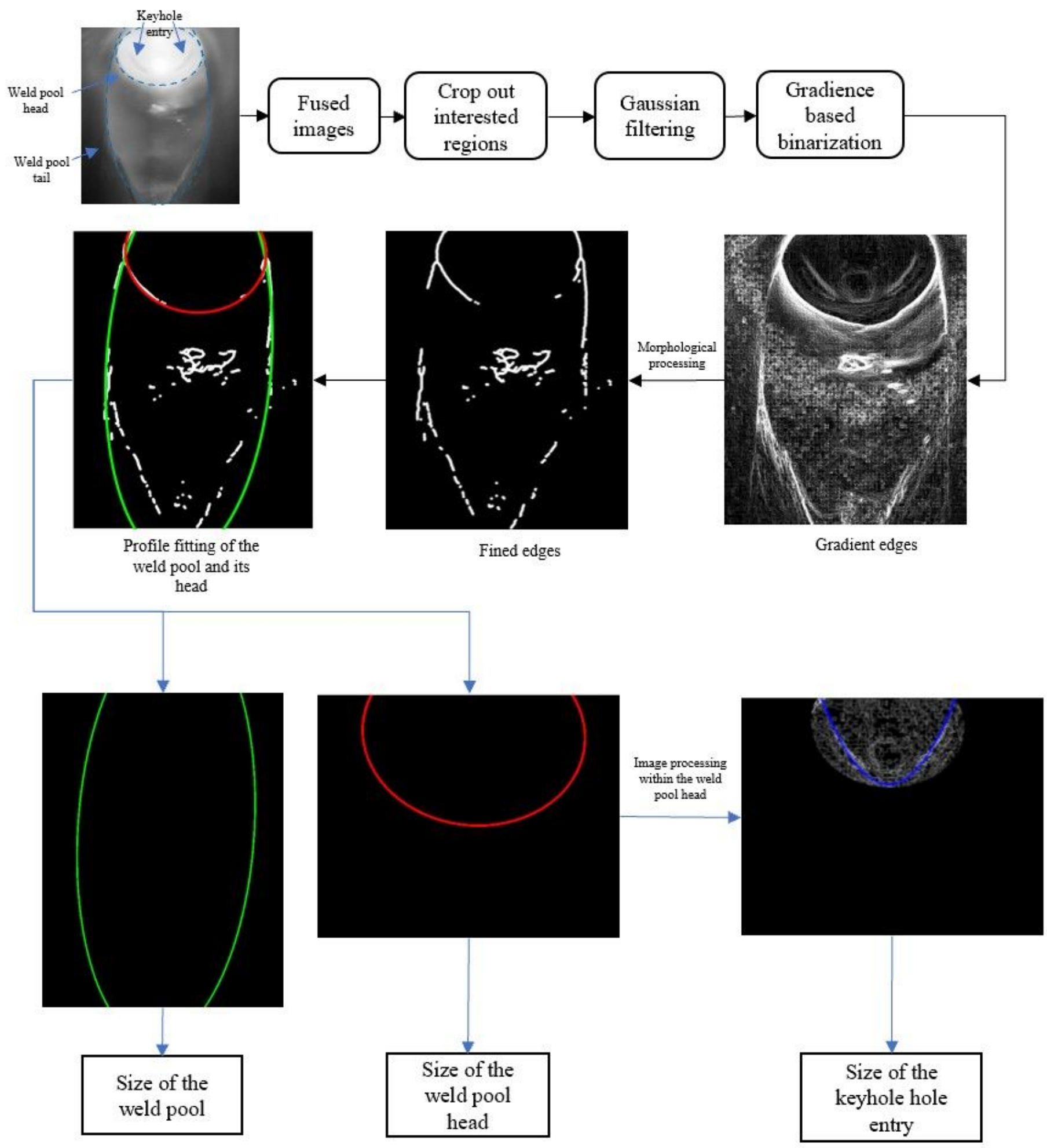

\section{Figure 9}

Extraction of the internal and external features of the weld pool based on adaptive image fusion. 


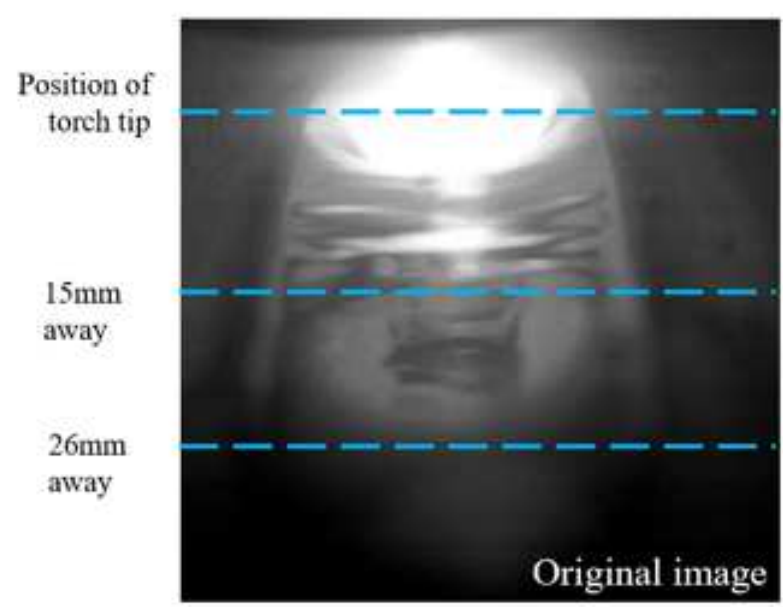

(a)

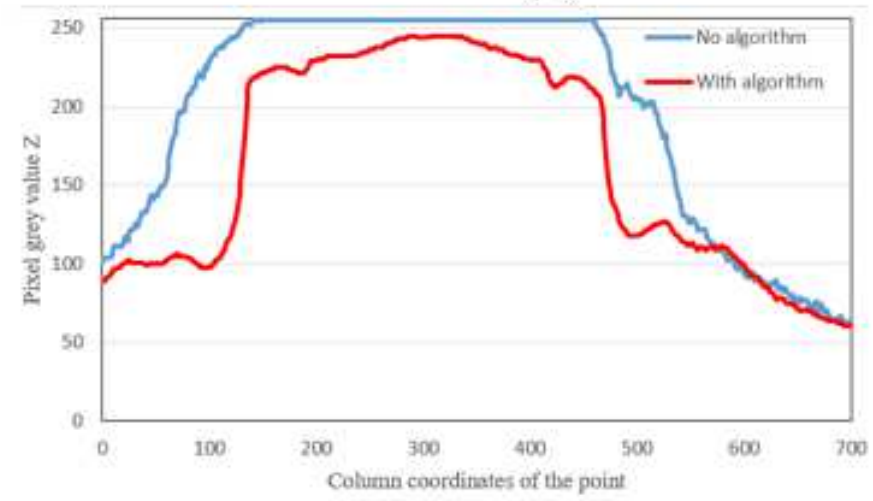

(c)

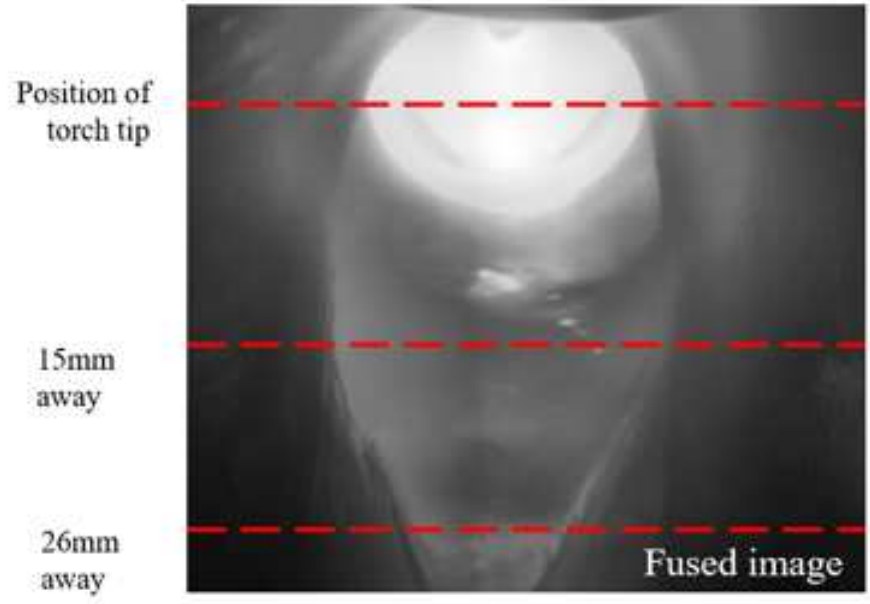

(b)

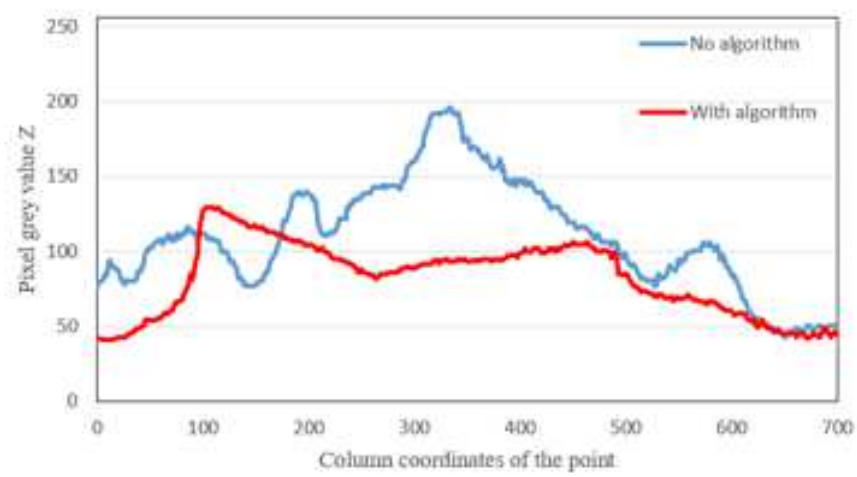

(d)

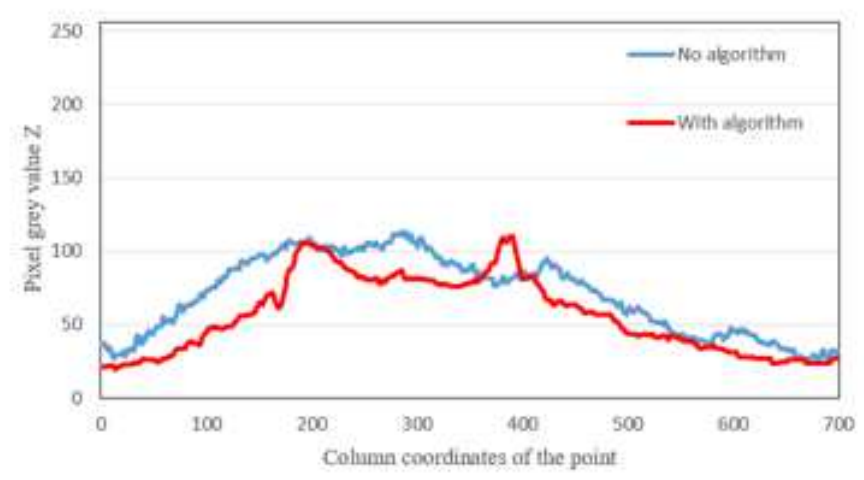

(e)

\section{Figure 10}

The comparison of the image pixel brightness histogram. (a)image without the algorithm, (b) image with the algorithm. 


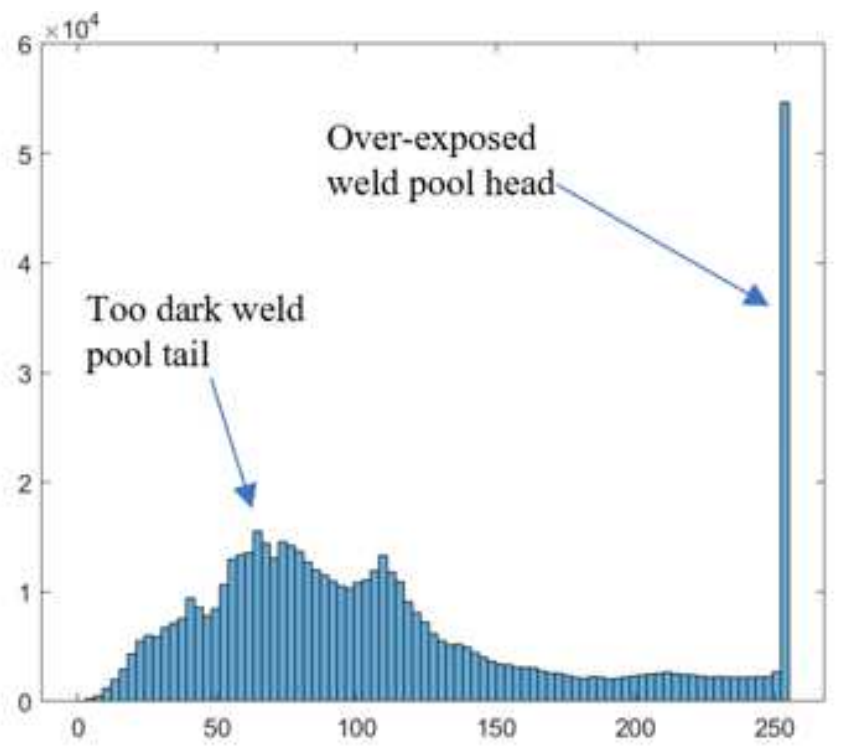

(a)

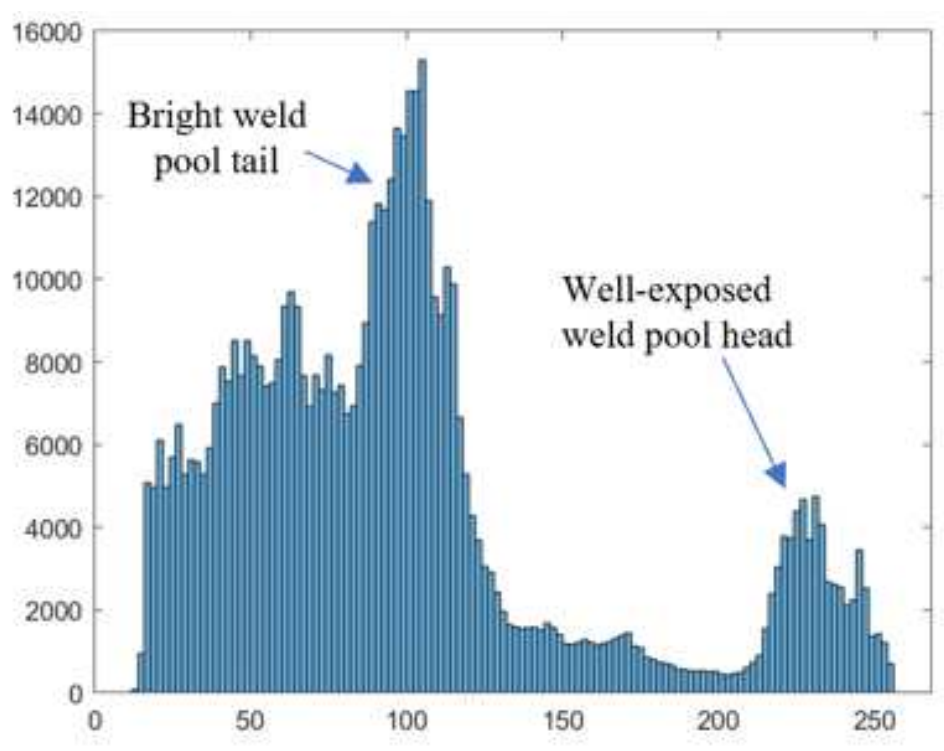

(b)

\section{Figure 11}

The improvement of the image with the proposed algorithm by comparison. (a)image without the algorithm, (b) image with the algorithm, (b) pixel values on the line below the torch tip, (d) pixel values on the line $15 \mathrm{~mm}$ away from the torch tip, (e) pixel values on the line $26 \mathrm{~mm}$ away from the torch tip.

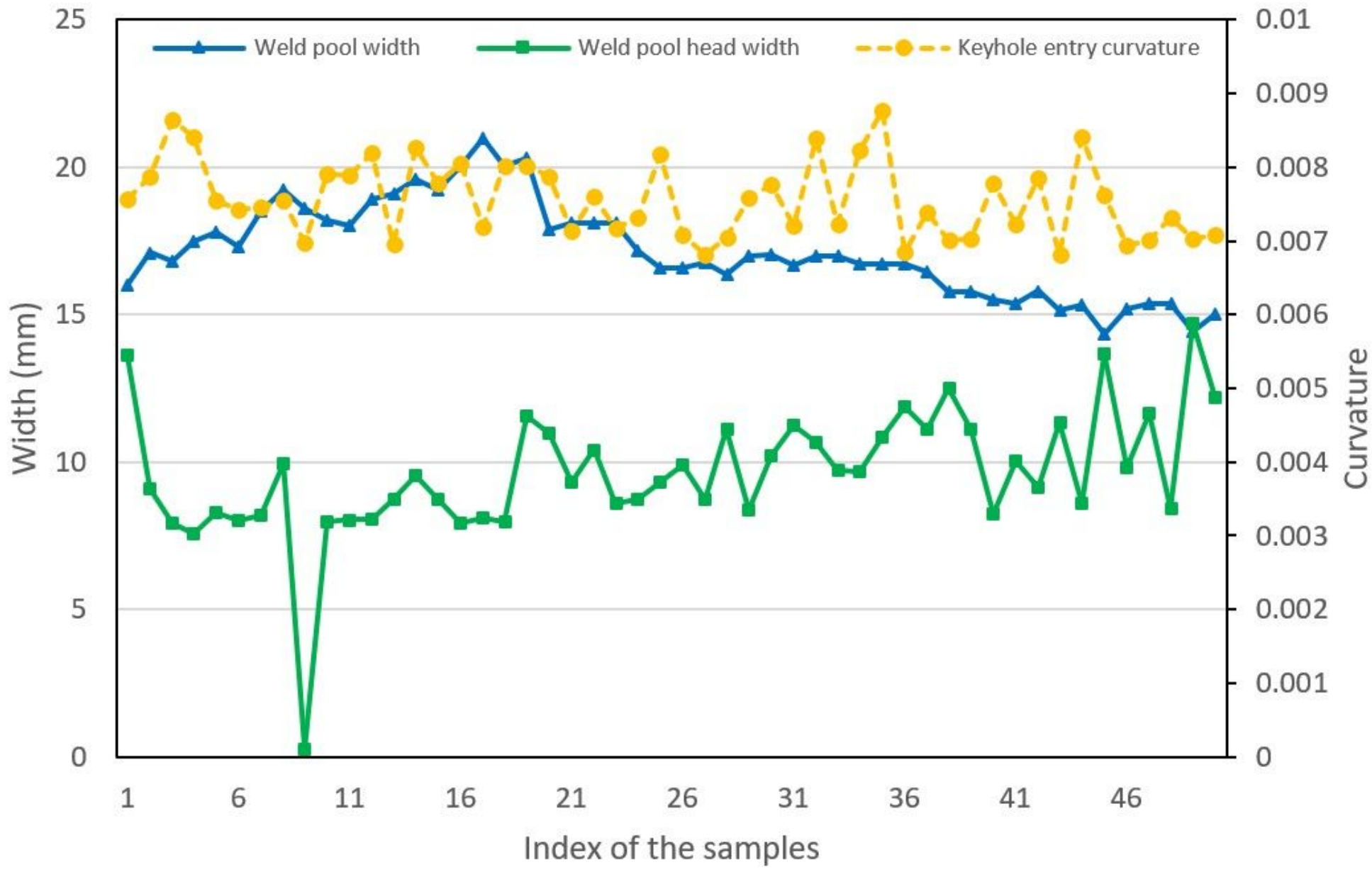

Figure 12 
Changes of the widths of the weld pool and its head and the curvature of the keyhole entry. 